$\exists \int$ Journal of Logic \& Analysis 5:2 (2013) 1-45

ISSN 1759-9008

\title{
Convergence in formal topology: a unifying notion
}

\author{
FRANCESCO CiRAULO \\ MARIA EMILIA MAIETTI \\ GIOVANNI SAMBIN
}

\begin{abstract}
Several variations on the definition of a Formal Topology exist in the literature. They differ on how they express convergence, the formal property corresponding to the fact that open subsets are closed under finite intersections. We introduce a general notion of convergence of which any previous definition is a special case. This leads to a predicative presentation and inductive generation of locales (formal covers), commutative quantales (convergent covers) and suplattices (basic covers) in a uniform way. Thanks to our abstract treatment of convergence, we are able to specify categorically the precise sense according to which our inductively generated structures are free, thus refining Johnstone's coverage theorem.

We also obtain a natural and predicative version of a fundamental result by Joyal and Tierney: convergent covers (commutative quantales) correspond to commutative co-semigroups over the category of basic covers (suplattices).
\end{abstract}

2010 Mathematics Subject Classification 54A05, 03F65 (primary); 06D22, 06F07, 06B23, 18B35 (secondary)

Keywords: formal topology, constructive mathematics

This paper aims to contribute to the development of constructive topology. By constructive topology we mean topology developed in a predicative and intuitionistic foundation. In order to avoid impredicative definitions the foundation must distinguish sets from collections; a typical example of a collection is given by all subsets of a set. The usual axiom of separation is then restricted to formulas that do not contain quantifications over collections. See Maietti and Sambin [18] and Maietti [17] for a formal system and for further explanations about such a foundation.

It is commonly accepted that, in order to develop topology constructively, the pointfree approach of locale theory by Johnstone [10] is the most convenient (Martin-Löf [21], Fourman and Grayson [9], Johnstone [11] and Sambin [29]). The predicative development of locale theory, started by Per Martin-Löf and the third author in [26], is now known as Formal Topology. To define a locale predicatively, one needs a base of opens that is a set, while the whole locale is only a collection (Curi [8]). The original notion 
of formal topology, proposed in [26], corresponds to that of open (or overt) locale (Joyal and Tierney [13] and Negri [24]). Here we call formal cover the generalization of formal topology corresponding to a locale; it is obtained by simply dropping the so-called positivity predicate in [26]. Because of the presence of bases, morphisms between formal covers/formal topologies are suitable relations. So they acquire a direct intuitive interpretation both in the direction of locales and in the opposite direction, namely that of frames [10].

Since its introduction in [26], the notion of formal topology has been presented in several different ways. One of the motivations was that of including relevant examples in a direct way, without artificial tricks. For instance, the original version in [26], or that in [29], works well for Stone spaces (Negri [23]), Scott domains, Zariski topologies,..., while the variant in Coquand [6] and in Coquand et al. [7] is more suitable for Baire spaces, algebraic domains, Kripke models and discrete topologies.

No variant superseded the others; actually, while in the general case they are all equivalent, they are no longer equivalent in the unary or finitary case. For example, one version of unary formal topologies represents Scott domains (Sambin, Valentini and Virgili [31]), while another algebraic domains (Sambin [28]).

All presentations of formal topology in the literature differ only in their way of expressing closure of opens under finite intersections, which in a pointfree approach appears as distributivity of finite meets over arbitrary joins in the lattice of opens. This property is here called convergence. In order to express convergence, in this paper we introduce a binary operation $\circ$ on subsets of the base with suitable conditions. We thus achieve a new unifying notion of formal cover/formal topology. All previous presentations are obtained as a special case by imposing some further conditions on the operation $\circ$.

Our definition gives a new predicative presentation of locales. This is obtained in a modular way (see table in section 4.2) starting from suplattices and passing through a new presentation of quantales. The constructive notion corresponding to suplattices is called basic cover by Sambin [29, 30]. It is thought of as a generalized pointfree topology without convergence. The category of basic covers is (impredicatively) dual to that of suplattices and hence it gives a genuine generalization of the category of locales.

By choosing to work in the direction of locale maps, the category of basic covers becomes the right setting to prove a predicative counterpart of Joyal-Tierney's result [13] stating that frames are special commutative monoids over the category of suplattices. We achieve this by introducing the notion of convergent cover, namely a basic cover 
equipped with a weak form of the operation $\circ$. The category of convergent covers is (impredicatively) dual to that of commutative quantales. ${ }^{1}$

By means of the new notions we can prove Joyal-Tierney's result in the following dualized form: convergent covers are commutative co-semigroups in the category of basic covers. A predicative proof of this is possible as soon as the tensor product exists predicatively, a fact which happens in the inductively generated case.

Just as a locale is a quantale in which multiplication and meet coincide, a formal cover is a convergent cover in which the operation $\circ$ corresponds to the lattice-theoretic meet. The category of formal covers is then (impredicatively) dual to that of locales.

The inclusion of all previous definitions in our new one leads to a unified, general method of inductive generation that applies to all variants. In particular, the rules of inductive generation, first given by Coquand et al. [7], become a special case of ours. In addition, we provide a method for generating also suplattices (basic covers) and quantales (convergent covers) in a modular way.

Thanks to the abstract character of our presentation of convergence, we are able to give a categorical reading of the inductive generation of formal covers and convergent covers. We can specify in what sense these constructions are free by showing that they provide object parts of adjoints to suitable forgetful functors. These results can be read as a refinement of Johnstone's coverage theorem [10] (see also Vickers [33]).

All the definitions and results of the present paper work equally well, with no modification, also when a positivity relation $\ltimes$ is added besides the cover as in Sambin $[29,30]$. In fact the addition of $\ltimes$, whose aim is to give a primitive pointfree version of closed subsets, does not affect the notion of convergence.

A general treatment of convergence as the one given here seems to be a necessary step towards a purely algebraic development of constructive topology. The present approach via the operation $\circ$ on subsets can be easily generalized to the algebraic framework basing on overlap algebras (for more on overlap algebras see Ciraulo and Sambin [5], Sambin [30] and Ciraulo, Maietti and Toto [2]).

\footnotetext{
${ }^{1}$ Actually, a constructive presentation of quantales is possible also using Sambin's notion of pretopology [27], but that presentation does not allow to prove Joyal-Tierney's result in a direct way.
} 


\section{Predicative suplattices: the notion of a basic cover}

The notion of basic cover recalled below can be read as a predicative topological presentation of a suplattice, or complete join-semilattice (Johnstone [10]). The corresponding notion of morphism makes the category of basic covers BCov dual (impredicatively) to that of suplattices and sup-preserving maps. This choice for the direction of arrows is justified by the fact that a suitable subcategory of BCov becomes equivalent to the category of locales; indeed, reaching a predicative version of locales in the context of basic covers is one of our aims.

We use the notation $Y \subseteq X$ to mean that $Y$ is a subset of $X$, where $X$ can be either a set or a collection. A subset in our foundation is defined by a propositional function with quantifications restricted to sets (see Maietti [17] and Sambin [30] for a more precise explanation). The collection of all subsets of a set $S$ is denoted by $\mathcal{P}(S)$.

Definition 1.1 Let $S$ be a set. A basic cover on $S$ is a relation $\triangleleft \subseteq S \times \mathcal{P}(S)$ between elements and subsets of $S$ that satisfies the following rules for every $a, b \in S$ and $U, V \subseteq S$ :

$$
\frac{a \epsilon U}{a \triangleleft U} \text { reflexivity } \quad \text { and } \quad \frac{a \triangleleft U \quad U \triangleleft V}{a \triangleleft V} \text { transitivity }
$$

where $U \triangleleft V \stackrel{\text { def }}{\Longleftrightarrow}(\forall b \in U)(b \triangleleft V)$.

Although this notion is pretty general, $S$ is often interpreted as a set of (names of) open subsets of a topology, typically a base. Then $a \triangleleft U$ is read: "the open subset (whose name is) $a$ is contained in the union of those belonging to $U$ ". For the sake of notation, we shall often confuse elements with singletons; for instance, we shall write $a \triangleleft b$ instead of $a \triangleleft\{b\}$, for $a, b \in S$. Moreover, we shall use the term "basic cover" also for the pair $(S, \triangleleft)$ itself.

For every basic cover $(S, \triangleleft)$ and every subset $U \subseteq S$, we put:

$$
\mathcal{A} U \stackrel{\text { def }}{=}\{a \in S \mid a \triangleleft U\} .
$$

This defines a saturation (or closure operator) on $\mathcal{P}(S)$, that is, a map $\mathcal{A}: \mathcal{P}(S) \rightarrow \mathcal{P}(S)$ which is monotone (with respect to inclusion), idempotent and expansive (that is, $U \subseteq \mathcal{A} U$ for all $U \subseteq S$ ). Vice versa, if $\mathcal{A}$ is a saturation on $\mathcal{P}(S)$, then $a \triangleleft U \stackrel{\text { def }}{\Leftrightarrow}$ $a \in \mathcal{A} U$ defines a basic cover on $S$. The correspondence between basic covers on $S$ and saturations on $\mathcal{P}(S)$ is a bijection (see Battilotti and Sambin [1] for details). 
Definition 1.2 For every basic cover $(S, \triangleleft)$ and every subset $U \subseteq S$, we say that $U$ is a formal open, or saturated, if $U=\mathcal{A} U$. We write $U={ }_{\mathcal{A}} V$ for $\mathcal{A} U=\mathcal{A} V$. The collection of all formal open subsets is written $\operatorname{Sat}(\mathcal{A})$.

Since $\mathcal{A}$ is idempotent, $\operatorname{Sat}(\mathcal{A})$ can be described also as the collection of all subsets of the form $\mathcal{A} U$, for $U \subseteq S$. Moreover, it is easy to see that $\operatorname{Sat}(\mathcal{A})$ can be identified with the quotient of $\mathcal{P}(S)$ modulo the equivalence relation ${ }_{\mathcal{A}}$.

It is well known that the collection of all fixed points of a saturation $\mathcal{A}$ can be given the structure of a suplattice. Joins are defined by:

$$
\bigvee_{i \in I}^{\mathcal{A}} \mathcal{A} W_{i} \stackrel{\text { def }}{=} \mathcal{A} \bigcup_{i \in I} \mathcal{A} W_{i}=\mathcal{A} \bigcup_{i \in I} W_{i}
$$

It is easy to see that the second equality holds; it says that $=_{\mathcal{A}}$ is respected by unions.

As a suplattice, $\operatorname{Sat}(\mathcal{A})$ is generated by the set-indexed family $\{\mathcal{A} a \mid a \in S\}$. Vice versa, if $\mathcal{L}$ is a suplattice which admits a set $S \subseteq \mathcal{L}$ of generators, ${ }^{2}$ then the structure $(S, \triangleleft)$, where $a \triangleleft U$ if $a \leq \bigvee U$, is a basic cover whose corresponding $\operatorname{Sat}(\mathcal{A})$ is isomorphic to $\mathcal{L}$. Thus, at least impredicatively, every suplattice is of the form $\operatorname{Sat}(\mathcal{A})$ (see [1] for details).

Impredicatively, every suplattice has a meet operation too. In the case of the suplattice $\operatorname{Sat}(\mathcal{A})$, meets exist also predicatively and are given by

$$
\mathcal{A} U \wedge^{\mathcal{A}} \mathcal{A} V=\mathcal{A}(\mathcal{A} U \cap \mathcal{A} V)=\mathcal{A} U \cap \mathcal{A} V .
$$

\subsection{Morphisms between basic covers}

For every binary relation $r$ between two sets $S$ and $T$, that is for every $r \subseteq S \times T$, as in Sambin [30] we define an operator $r^{-}: \mathcal{P}(T) \longrightarrow \mathcal{P}(S)$ by putting

$$
a \epsilon r^{-} V \Longleftrightarrow(\exists b \in V)(a r b)
$$

for every $a \in S$ and $V \subseteq T$.

Definition 1.3 Let $\mathcal{S}=(S, \triangleleft \mathcal{S})$ and $\mathcal{T}=(T, \triangleleft \mathcal{T})$ be two basic covers. A relation $r$ between $S$ and $T$ is a basic cover map, or it is said to respect covers, if:

$$
b \triangleleft_{\mathcal{T}} V \Longrightarrow r^{-} b \triangleleft_{\mathcal{S}} r^{-} V
$$

for every $b \in T$ and $V \subseteq T$. Two basic cover maps $r_{1}$ and $r_{2}$ from $\mathcal{S}$ to $\mathcal{T}$ are declared equal if $r_{1}{ }^{-} b={ }_{\mathcal{A}_{\mathcal{S}}} r_{2}{ }^{-} b$ for all $b \in T$. $^{3}$

\footnotetext{
${ }^{2}$ This requirement is not trivial since, predicatively, suplattices are not sets (see Curi [8]).

${ }^{3}$ Thus, properly speaking, a morphism between two basic covers is an equivalence class of relations satisfying (5).
} 
It is possible to show (see Sambin [30]) that equation (5) is exactly what is needed to make the assignment $\mathcal{A}_{\mathcal{T}} V \longmapsto \mathcal{A}_{\mathcal{S}} r^{-} V$ a well-defined sup-preserving map from $\operatorname{Sat}\left(\mathcal{A}_{\mathcal{T}}\right)$ to $\operatorname{Sat}\left(\mathcal{A}_{\mathcal{S}}\right)$. Vice versa, each sup-preserving map $h: \operatorname{Sat}\left(\mathcal{A}_{\mathcal{T}}\right) \longrightarrow \operatorname{Sat}\left(\mathcal{A}_{\mathcal{S}}\right)$ can be obtained in this way. In fact, it corresponds to the relation $r$ between $S$ and $T$ defined by $a r b$ if $a \in h\left(\mathcal{A}_{\mathcal{T}} b\right)$. One can see that two relations $r_{1}$ and $r_{2}$ are equal as basic cover maps exactly when $r_{1}{ }^{-} V={ }_{\mathcal{A}_{\mathcal{S}}} r_{2}{ }^{-} V$ for all $V \subseteq T$, that is, exactly when they correspond to the same map between suplattices.

Proposition 1.4 Basic covers and basic cover maps (modulo their equality) form a category, called BCov. Identities are represented by (the class of) identity relations. Composition is usual composition of relations.

The category BCov is (impredicatively) dual to the category SupLat of suplattices and sup-preserving maps.

Proof It is straightforward to show that BCov is a category. The previous discussion shows that it is dual to SupLat (see Battilotti and Sambin [1] for more details).

\subsection{Inductive generation of basic covers}

Coquand et al. [7] describe a method for inductively generating basic covers and give a predicative justification for it. Namely, they construct a basic cover satisfying arbitrary axioms of the form $a \triangleleft U$. The problem is that simply taking the reflexive and transitive (in the sense of definition 1.1) closure of the axioms is not a well-founded procedure from a predicative point of view. In fact, accepting transitivity as an inductive rule requires to consider a collection of assumptions, one for each subset $U$ in the rule. So, an impredicative argument is necessary to get a fixed point of the operator associated with the inductive clauses. This is confirmed by the fact that some formal topologies cannot be inductively generated (see [7]).

In [7] it is shown how to solve this problem. Given a set $S$, one needs a set-indexed family of axioms of the form $a \triangleleft U$. This means that one has a set $I(a)$ for each $a \in S$ and, for each $a \in S$ and $i \in I(A)$, a subset $C(a, i) \subseteq S$ with the intended meaning that $a \triangleleft C(a, i)$ holds. The pair $I, C$ is called an axiom-set.

With every axiom-set $I, C$ one can associate a basic cover, say $\triangleleft_{I, C}$, such that:

(i) $a \triangleleft_{I, C} C(a, i)$ for every $a \in S$ and $i \in I(a)$;

(ii) if $\triangleleft^{\prime}$ is another basic cover such that $a \triangleleft^{\prime} C(a, i)$ for all $a \in S$ and $i \in I(A)$, then $a \triangleleft_{I, C} U \Rightarrow a \triangleleft^{\prime} U$ for all $a \in S$ and $U \subseteq S$. 
In other words, $\triangleleft_{I, C}$ is the least cover satisfying the axioms $a \triangleleft_{I, C} C(a, i)$ for all $a \in S$ and $i \in I(a)$. One can show (see [7]) that $\triangleleft_{I, C}$ is the unique relation between elements and subsets of $S$ which satisfies:

i. $\frac{a \epsilon U}{a \triangleleft U}$ reflexivity;

ii. $\frac{i \in I(a) \quad C(a, i) \triangleleft U}{a \triangleleft U}$ infinity (transitivity restricted to axioms);

iii. induction: for every $P \subseteq S$, if $P$ satisfies

$$
\frac{b \in U}{b \in P} \quad \text { and } \quad \frac{i \in I(b) \quad C(b, i) \subseteq P}{b \in P} \quad \text { for all } b \in S
$$

then $a \triangleleft U$ implies $a \in P$;

for all $a \in S$ and $U \subseteq S$.

As recalled in [7], this kind of inductive definition can be formalized and justified in a constructive framework such as Martin-Löf type theory. In practice, proving $a \triangleleft U \rightarrow a \epsilon P$ by induction on $a \triangleleft U$ means checking that $a \epsilon P$ holds in either of the two cases: the assumption $a \in U$ and the inductive hypothesis $C(a, i) \subseteq P$ for some $i \in I(a)$.

Definition 1.5 A basic cover $(S, \triangleleft)$ is inductively generated if there exists an axiomset $I, C$ such that $\triangleleft=\triangleleft_{I, C}$, that is, $\triangleleft$ satisfies the rules above.

We call $\mathbf{B C o v}_{i}$ the full subcategory of $\mathbf{B C o v}$ whose objects are inductively generated.

We recall from [7] that the category $\mathbf{B C o v}_{i}$ of inductively generated basic cover is predicatively a proper subcategory of $\mathbf{B C o v}$, since there are examples of basic covers (actually formal covers!) that cannot be inductively generated.

When restricting to inductively generated basic covers, a relation is a basic cover map if it respects the axioms in the following sense:

Lemma 1.6 Let $\mathcal{S}=\left(S, \triangleleft_{\mathcal{S}}\right)$ and $\mathcal{T}=\left(T, \triangleleft_{\mathcal{T}}\right)$ be two basic covers and let $r$ be a relation between $S$ and $T$. If $\mathcal{T}$ is inductively generated by the axiom-set $I, C$, then $r$ is a basic cover map from $\mathcal{S}$ to $\mathcal{T}$ if and only if $r^{-} b \triangleleft_{\mathcal{S}} r^{-} C(b, i)$ holds for all $b \in T$ and all $i \in I(b)$.

Proof If $r$ is a basic cover map, then for all $b \in T$ and $i \in I(b)$ one has $r^{-} b \triangleleft_{\mathcal{S}}$ $r^{-} C(b, i)$ because $b \triangleleft_{\mathcal{T}} C(b, i)$. Vice versa, we prove that $b \triangleleft_{\mathcal{T}} V \Rightarrow r^{-} b \triangleleft_{\mathcal{S}} r^{-} V$ for 
all $b \in T$ and $V \subseteq T$ by induction on the generation of $b \triangleleft_{\mathcal{T}} V$. If $b \triangleleft_{\mathcal{T}} V$ follows by reflexivity, then from $b \in V$ we have $r^{-} b \subseteq r^{-} V$ and hence $r^{-} b \triangleleft_{\mathcal{S}} r^{-} V$ by reflexivity of $\triangleleft_{\mathcal{S}}$. If $b \triangleleft_{\mathcal{T}} V$ follows by infinity, then we have $C(b, i) \triangleleft \mathcal{T} V$ for some $i \in I(b)$. By the inductive hypothesis applied to all elements of $C(b, i)$ we get $r^{-} C(b, i) \triangleleft_{\mathcal{S}} r^{-} V$. This, together with the assumption, gives $r^{-} b \triangleleft_{\mathcal{S}} r^{-} V$ (by transitivity of $\triangleleft_{\mathcal{S}}$ ).

Impredicatively, every basic cover $(S, \triangleleft)$ can be generated by means of an axiom-set $I, C$, where $I(a)=\{U \subseteq S \mid a \triangleleft U\}$ for every $a \in S$ and $C(a, U)=U$ for $U \in I(A)$. So $\mathbf{B C o v}_{i}$ and $\mathbf{B C o v}$ coincide impredicatively.

Recall from Mac Lane [15, chapter VII] that a symmetric monoidal category is given by the following data: a category $C$; a notion of "tensor product", that is, a bifunctor $\otimes: C \times C \rightarrow C$; a unit object $E$; and four natural isomorphisms

$$
\begin{aligned}
& \alpha_{\mathcal{S}_{1}, \mathcal{S}_{2}, \mathcal{S}_{3}}: \mathcal{S}_{1} \otimes\left(\mathcal{S}_{2} \otimes \mathcal{S}_{3}\right) \\
& \lambda_{\mathcal{S}}: E \otimes \mathcal{S} \longrightarrow \mathcal{S} \quad \longrightarrow \quad\left(\mathcal{S}_{1} \otimes \mathcal{S}_{2}\right) \otimes \mathcal{S}_{3} \\
& \gamma_{\mathcal{S}_{1}, \mathcal{S}_{2}}: \mathcal{S}_{1} \otimes \mathcal{S}_{2} \longrightarrow \mathcal{S}_{2} \otimes \mathcal{S} \otimes E \longrightarrow \mathcal{S}
\end{aligned}
$$

(with $\mathcal{S}, \mathcal{S}_{1}, \ldots, \mathcal{S}_{4}$ objects of $C$ ) which satisfy the following coherence conditions:

$$
\begin{aligned}
& \alpha_{\mathcal{S}_{1} \otimes \mathcal{S}_{2}, \mathcal{S}_{3}, \mathcal{S}_{4}} \cdot \alpha_{\mathcal{S}_{1}, \mathcal{S}_{2}, \mathcal{S}_{3} \otimes \mathcal{S}_{4}}=\left(\alpha_{\mathcal{S}_{1}, \mathcal{S}_{2}, \mathcal{S}_{3}} \otimes i d_{\mathcal{S}_{4}}\right) \cdot \alpha_{\mathcal{S}_{1}, \mathcal{S}_{2} \otimes \mathcal{S}_{3}, \mathcal{S}_{4}} \cdot\left(i d_{\mathcal{S}_{1}} \otimes \alpha_{\mathcal{S}_{2}, \mathcal{S}_{3}, \mathcal{S}_{4}}\right), \\
& \left(\rho_{\mathcal{S}_{1}} \otimes i d_{\mathcal{S}_{2}}\right) \cdot \alpha_{\mathcal{S}_{1}, E, \mathcal{S}_{2}}=i d_{\mathcal{S}_{1}} \otimes \lambda_{\mathcal{S}_{2}}, \quad \lambda_{E}=\rho_{E}, \\
& \gamma_{\mathcal{S}_{2}, \mathcal{S}_{1}}=\gamma_{\mathcal{S}_{1}, \mathcal{S}_{2}}{ }^{-1}, \quad \rho_{\mathcal{S}}=\lambda_{\mathcal{S}} \cdot \gamma_{\mathcal{S}, E}, \\
& \alpha_{\mathcal{S}_{3}, \mathcal{S}_{1}, \mathcal{S}_{2}} \cdot \gamma_{\mathcal{S}_{1} \otimes \mathcal{S}_{2}, \mathcal{S}_{3}} \cdot \alpha_{\mathcal{S}_{1}, \mathcal{S}_{2}, \mathcal{S}_{3}}=\left(\gamma_{\mathcal{S}_{1}, \mathcal{S}_{3}} \otimes i d_{\mathcal{S}_{2}}\right) \cdot \alpha_{\mathcal{S}_{1}, \mathcal{S}_{3}, \mathcal{S}_{2}} \cdot\left(i d_{\mathcal{S}_{1}} \otimes \gamma_{\mathcal{S}_{2}, \mathcal{S}_{3}}\right)
\end{aligned}
$$

(actually $\lambda_{E}=\rho_{E}$ and $\rho_{\mathcal{S}}=\lambda_{\mathcal{S}} \cdot \gamma_{\mathcal{S}, E}$ are redundant by Kelly [14]).

Proposition 1.7 $\mathbf{B C o v}_{i}$ is a symmetric monoidal category.

Proof Let $\mathcal{S}$ and $\mathcal{T}$ be two basic covers inductively generated by the axiom-sets $I, C$ and $J, D$, respectively. The tensor $\mathcal{S} \otimes \mathcal{T}$ is the basic cover $\left(S \times T, \triangleleft_{\mathcal{S} \otimes \mathcal{T}}\right)$ generated by the axioms

$$
(a, b) \triangleleft_{\mathcal{S} \otimes \mathcal{T}} C(a, i) \times\{b\} \quad \text { and } \quad(a, b) \triangleleft_{\mathcal{S} \otimes \mathcal{T}}\{a\} \times D(b, j)
$$

for every $(a, b) \in S \times T$ and every $i \in I(a)$ and $j \in J(b)$. This definition, which follows the same idea as in [7, section 4.3], is a predicative rendering of the construction of the the tensor product by Joyal and Tierney [13] as presented by Vickers and Johnstone [12]. We leave the details showing that this is indeed an axiom-set. 
For every pair $\left(r_{1}, r_{2}\right)$ of morphisms in $\mathbf{B C o v}_{i}$, with $r_{1}: \mathcal{S}_{1} \longrightarrow \mathcal{T}_{1}$ and $r_{2}: \mathcal{S}_{2} \longrightarrow$ $\mathcal{T}_{2}$, the tensor $r_{1} \otimes r_{2}$ is by definition the unique morphism from $\mathcal{S}_{1} \otimes \mathcal{S}_{2}$ to $\mathcal{T}_{1} \otimes \mathcal{T}_{2}$ such that $\left(r_{1} \otimes r_{2}\right)^{-}\left(b_{1}, b_{2}\right)={ }_{\mathcal{A}_{\mathcal{S}_{1} \otimes \mathcal{S}_{2}}}\left(r_{1}{ }^{-} b_{1}\right) \times\left(r_{2}{ }^{-} b_{2}\right)$ for all $b_{1}, b_{2}$.

The unit $E$ of the tensor is given by the cover generated on the singleton set $\{*\}$ by means of no axioms. The due isomorphisms are defined in the obvious way. For instance, $\gamma_{\mathcal{S}_{1}, \mathcal{S}_{2}}$ is the unique basic cover map (up to equality) such that $\gamma_{\mathcal{S}_{1}, \mathcal{S}_{2}}{ }^{-}\left(a_{2}, a_{1}\right)$ $=\mathcal{A}_{\mathcal{S}_{1} \otimes \mathcal{S}_{2}}\left(a_{1}, a_{2}\right)$. Similarly, $\alpha$ is defined by $\alpha_{\mathcal{S}_{1}, \mathcal{S}_{2}, \mathcal{S}_{3}}{ }^{-}\left(\left(a_{1}, a_{2}\right), a_{3}\right)=\mathcal{A}_{\left(\mathcal{S}_{1} \otimes \mathcal{S}_{2}\right) \otimes \mathcal{S}_{3}}$ $\left(a_{1},\left(a_{2}, a_{3}\right)\right)$. It is easy to check that $\alpha, \rho, \lambda$ and $\gamma$ are all natural and satisfy the required coherence conditions.

One can prove that the tensor functor on a basic cover $\mathcal{S}$

$$
\mathcal{S} \otimes(-): \mathbf{B C o v}_{\mathbf{i}} \longrightarrow \mathbf{B C o v}_{\mathbf{i}}
$$

has impredicatively the left adjoint

$$
(-) \rightarrow \mathcal{S}: \mathbf{B C o v}_{\mathbf{i}} \longrightarrow \mathbf{B C o v}_{\mathbf{i}}
$$

where $\mathcal{T} \rightarrow \mathcal{S}$ is the impredicative basic cover corresponding to the suplattice of all basic cover maps from $\mathcal{T}$ to $\mathcal{S}$ ordered pointwise:

$$
r \leq s \equiv(\forall U \in \mathcal{P}(S)) r^{-} U \triangleleft s^{-} U
$$

(a predicative treatment of exponentiation is known only in a more restricted setting where the above tensor becomes a product, see Maietti [16] and Maietti and Valentini [20]). In other words, $\mathcal{S} \otimes(-)$ as a functor on $\mathbf{B C o v}_{\mathbf{i}}{ }^{o p}$ has a right adjoint. Therefore the tensor on $\mathbf{B} \mathbf{C o v}_{i}$, which is impredicatively the opposite of the category SupLat, coincides with the Galois tensor defined by Joyal-Tierney in [13] (see also Johnstone and Vickers [12]).

The above definition of $\otimes$ benefits from a topological intuition. Indeed, the following lemma shows that the basic cover of $\mathcal{S} \otimes \mathcal{T}$ satisfies an analogue of a key property of the product of two topological spaces.

Lemma 1.8 Let $\mathcal{S}$ and $\mathcal{T}$ be two inductively generated basic covers. Then

$$
\frac{a \triangleleft_{\mathcal{S}} U \quad b \triangleleft_{\mathcal{T}} V}{(a, b) \triangleleft \mathcal{S} \otimes \mathcal{T} U \times V}
$$

holds for all $a \in S, b \in T, U \subseteq S$ and $V \subseteq T$.

Proof By double induction on the proofs of $a \triangleleft_{\mathcal{S}} U$ and $b \triangleleft_{\mathcal{T}} V$. We must analyze four different cases: $a \in U$ and $b \in V ; a \in U$ and $D(b, j) \triangleleft \mathcal{T} V$ for some $j \in J(b)$; 
$C(a, i) \triangleleft_{\mathcal{S}} U$ for some $i \in I(a)$ and $b \in V ; C(a, i) \triangleleft_{\mathcal{S}} U$ for some $i \in I(a)$ and $D(b, j) \triangleleft_{\mathcal{T}} V$ for some $j \in J(b)$. All cases are proved similarly. For instance, let $a \in U$ and $D(b, j) \triangleleft_{\mathcal{T}} V$ for some $j \in J(b)$, that is $b^{\prime} \triangleleft_{\mathcal{T}} V$ for all $b^{\prime} \in D(b, j)$. Then by inductive hypothesis we get $\left(a, b^{\prime}\right) \triangleleft_{\mathcal{S} \otimes \mathcal{T}} U \times V$ for all $b^{\prime} \in D(b, j)$, that is $\{a\} \times D(b, j) \triangleleft_{\mathcal{S} \otimes \mathcal{T}} U \times V$. Hence $(a, b) \triangleleft_{\mathcal{S} \otimes \mathcal{T}} U \times V$ by (6).

This lemma can be expressed as $\mathcal{A}_{\mathcal{S}} U \times \mathcal{A}_{\mathcal{T}} V \triangleleft_{\mathcal{S} \otimes \mathcal{T}} U \times V$ and hence also as the equation

$$
\mathcal{A}_{\mathcal{S}} U \times \mathcal{A}_{\mathcal{T}} V=\mathcal{A}_{\mathcal{S} \otimes \mathcal{T}} U \times V
$$

since $U \times V \triangleleft_{\mathcal{S} \otimes \mathcal{T}} \mathcal{A}_{\mathcal{S}} U \times \mathcal{A}_{\mathcal{T}} V$ holds by reflexivity.

\section{Operations on formal opens}

A locale is a suplattice in which binary meets distribute over arbitrary joins. Since our aims include the inductive generation of locales, we wish to modify the inductive generation of a basic cover $\mathcal{A}$ so that the resulting lattice $\operatorname{Sat}(\mathcal{A})$ (recall that by (3) it always has a meet) satisfies distributivity. The mere requirement of distributivity of $\operatorname{Sat}(\mathcal{A})$ says nothing on how to obtain it when $\triangleleft$ is generated inductively. As we will see, however, it is possible to impose distributivity by adding an extra primitive operation $\circ$ on subsets of the base $S$ with certain suitable properties. In fact, using $\circ$ one can impose some conditions during the generation process which guarantee that distributivity holds "at the end", when the generation of $\triangleleft$ is "completed".

This method extends in a natural way to the generation of quantales. Recall that a quantale is a suplattice with an associative binary operation, called multiplication, that is distributive over joins (Rosenthal [25]). The idea is to make $\operatorname{Sat}(\mathcal{A})$ a quantale $\left(\operatorname{Sat}(\mathcal{A}), \bigvee^{\mathcal{A}},{ }^{\mathcal{A}}\right)$ where the multiplication $\circ^{\mathcal{A}}$ is induced by an operation $\circ$ on subsets of $S$. In this section we see what conditions on $\circ$ make $\circ^{\mathcal{A}}$ well-defined, commutative and associative in $\operatorname{Sat}(\mathcal{A})$. In the next section we will study the case of distributivity of $\circ^{\mathcal{A}}$ and later the special case of locales.

We start by specifying how an operation ${ }^{\mathcal{A}}$ on $\operatorname{Sat}(\mathcal{A})$ is obtained in terms of a given operation $\circ$ on $\mathcal{P}(S)$. Our heuristic criterion is to read an element $\mathcal{A} U$ of $\operatorname{Sat}(\mathcal{A})$ as an ideal object which is approximated by the concrete subset $U$. This view is suggested by the case in which $\mathcal{A}$ is inductively generated and thus $\mathcal{A} U$ is only the "limit" of the generation process. Then it is natural to require that the operation ${ }^{\mathcal{A}}$ on $\operatorname{Sat}(\mathcal{A})$ is approximated by the operation $\circ$ on $\mathcal{P}(S)$. Thus we put

$$
\mathcal{A} U \circ \mathcal{A} \mathcal{A} V=\mathcal{A}(U \circ V)
$$


which says that applying $\circ$ to approximations $U$ of $\mathcal{A} U$ and $V$ of $\mathcal{A} V$ produces an approximation $U \circ V$ of $\mathcal{A} U \circ{ }^{\mathcal{A}} \mathcal{A} V$. This equation is our starting point to find the right conditions on the operation $\odot$. First of all, in order to read equation (8) as the definition of $\circ^{\mathcal{A}}$, we must understand what conditions on $\circ$ make $\circ^{\mathcal{A}}$ well-defined.

Proposition 2.1 For every basic cover $(S, \triangleleft)$ and every binary operation $\circ$ on $\mathcal{P}(S)$, the following are equivalent:

(1) $\circ^{\mathcal{A}}$ as defined in $(8)$ is a well-defined operation on $\operatorname{Sat}(\mathcal{A})$, that is:

$$
\left(\mathcal{A} U=\mathcal{A} U^{\prime}\right) \&\left(\mathcal{A} V=\mathcal{A} V^{\prime}\right) \Longrightarrow \mathcal{A}(U \circ V)=\mathcal{A}\left(U^{\prime} \circ V^{\prime}\right)
$$

for all $U, U^{\prime}, V, V^{\prime} \subseteq S$ (in other words, o respects $=_{\mathcal{A}}$ );

(2) $\mathcal{A}(\mathcal{A} U \circ \mathcal{A} V)=\mathcal{A}(U \circ V)$, for all $U, V \subseteq S$.

Proof $(1 \Rightarrow 2)$ Since $\mathcal{A}$ is idempotent, $\mathcal{A} \mathcal{A} U=\mathcal{A} U$ and $\mathcal{A} \mathcal{A} V=\mathcal{A} V$ hold and then $\mathcal{A}(\mathcal{A} U \circ \mathcal{A} V)=\mathcal{A}(U \circ V)$, that is, 2 .

(2 $\Rightarrow 1$ ) Assume $\mathcal{A} U=\mathcal{A} U^{\prime}$ and $\mathcal{A} V=\mathcal{A} V^{\prime}$. Then $\mathcal{A} U \circ \mathcal{A} V=\mathcal{A} U^{\prime} \circ \mathcal{A} V^{\prime}$ and hence, a fortiori, $\mathcal{A}(\mathcal{A} U \circ \mathcal{A} V)=\mathcal{A}\left(\mathcal{A} U^{\prime} \circ \mathcal{A} V^{\prime}\right)$. By 2, this gives $\mathcal{A}(U \circ V)=\mathcal{A}\left(U^{\prime} \circ V^{\prime}\right)$. So 1 is proved.

Assuming (8), item 2 above says that

$$
\mathcal{A} U \circ{ }^{\mathcal{A}} \mathcal{A} V=\mathcal{A}(\mathcal{A} U \circ \mathcal{A} V)
$$

If one takes this equation as a definition of ${ }^{\mathcal{A}}$, one immediately obtains a well-defined operation on $\operatorname{Sat}(\mathcal{A})$ without extra requirements for $\odot$. Nevertheless, we have not done like that since (9) does not satisfy our intuition on approximations. In fact, (9) is of no use when $\mathcal{A}$ is inductively generated since it produces an approximation of $\mathcal{A} U \circ{ }^{\mathcal{A}} \mathcal{A} V$ only "after" the generation of the ideal objects $\mathcal{A} U$ and $\mathcal{A} V$ is "completed".

Lemma 2.2 Let $(S, \triangleleft)$ be a basic cover and let $\circ$ be a binary operation on $\mathcal{P}(S)$. Then the following are equivalent:

(1) $\frac{U \triangleleft V}{U \circ W \triangleleft V \circ W}$ and $\frac{U \triangleleft V}{W \circ U \triangleleft W \circ V}$ localization;

(2) $\frac{U_{1} \triangleleft V_{1} \quad U_{2} \triangleleft V_{2}}{U_{1} \circ U_{2} \triangleleft V_{1} \circ V_{2}} \quad$ stability;

(3) ${ }^{\mathcal{A}}$ as defined in (8) respects inclusion, that is:

$$
\left(\mathcal{A} U_{1} \subseteq \mathcal{A} V_{1}\right) \&\left(\mathcal{A} U_{2} \subseteq \mathcal{A} V_{2}\right) \Longrightarrow\left(\mathcal{A} U_{1} \circ \mathcal{A} \mathcal{A} U_{2}\right) \subseteq\left(\mathcal{A} V_{1} \circ^{\mathcal{A}} \mathcal{A} V_{2}\right)
$$

for all $U_{1}, U_{2}, V_{1}, V_{2} \subseteq S$. 
Proof (1. $\Leftrightarrow 2$.) Assume localization. If $U_{1} \triangleleft V_{1}$ and $U_{2} \triangleleft V_{2}$, then both $U_{1} \circ U_{2} \triangleleft$ $V_{1} \circ U_{2}$ and $V_{1} \circ U_{2} \triangleleft V_{1} \circ V_{2}$ hold; hence $U_{1} \circ U_{2} \triangleleft V_{1} \circ V_{2}$ by transitivity. Vice versa, the rules of localization are particular cases of stability, since $W \triangleleft W$.

(2. $\Leftrightarrow 3$.) Recall that $U \triangleleft V$ iff $\mathcal{A} U \subseteq \mathcal{A} V$. So 2 can be rewritten as $\mathcal{A} U_{1} \subseteq \mathcal{A} V_{1} \&$ $\mathcal{A} U_{2} \subseteq \mathcal{A} V_{2} \Rightarrow \mathcal{A}\left(U_{1} \circ U_{2}\right) \subseteq \mathcal{A}\left(V_{1} \circ V_{2}\right)$ that is 3 by definition of $\circ^{\mathcal{A}}$.

Item 3, together with (8), implies that $\circ$ respects $=_{\mathcal{A}}$. So each of the three items above is a sufficient condition for $\circ^{\mathcal{A}}$ to be well-defined. This is not surprising since they express monotonicity of $\circ$ with respect to the preorder induced by $\triangleleft$ on $\mathcal{P}(S)$, and $=_{\mathcal{A}}$ is the equivalence relation associated with it.

Most properties one can require on $\circ^{\mathcal{A}}$ are induced in a natural way by corresponding properties linking $\circ$ with the cover. For instance, ${ }^{\mathcal{A}}$ is commutative iff $\mathcal{A}(U \circ V)=$ $\mathcal{A}(V \circ U)$, that is $U \circ V={ }_{\mathcal{A}} V \circ U$. Similarly, ${ }^{\mathcal{A}}$ is associative iff $\circ$ is associative modulo $=_{\mathcal{A}}$, that is $(U \circ V) \circ W={ }_{\mathcal{A}} U \circ(V \circ W)$, for all $U, V, W \subseteq S$. In this paper, for simplicity's sake, we shall always assume $\circ^{\mathcal{A}}$ to be associative and commutative.

For future reference, it is convenient to fix a name for a basic cover with an operation $\circ$ such that $\circ^{\mathcal{A}}$ is well-defined, monotone, associative and commutative. Thanks to the previous lemma, the definition can be reduced to the following form.

Definition 2.3 We say that $(S, \triangleleft, \circ)$ is a basic cover with operation if $\mathcal{S}=(S, \triangleleft)$ is a basic cover and $\circ$ is a binary operation on $\mathcal{P}(S)$ which satisfies:

(1) $\frac{U_{1} \triangleleft V_{1} \quad U_{2} \triangleleft V_{2}}{U_{1} \circ U_{2} \triangleleft V_{1} \circ V_{2}} \quad$ stability; or, equivalently, localization: $\frac{U \triangleleft V}{U \circ W \triangleleft V \circ W}$;

(2) $(U \circ V) \circ W \triangleleft U \circ(V \circ W)$ associativity with respect to $=_{\mathcal{A}}$;

(3) $U \circ V \triangleleft V \circ U$ commutativity with respect to $=_{\mathcal{A}}$.

In this case, the equation $\mathcal{A} U \circ \mathcal{A} \mathcal{A} V \stackrel{\text { def }}{=} \mathcal{A}(U \circ V)$ defines a monotone, associative and commutative operation on the suplattice $\operatorname{Sat}(\mathcal{A})$.

Thanks to stability, ${ }^{\mathcal{A}}$ is a map $\operatorname{Sat}(\mathcal{A}) \times \operatorname{Sat}(\mathcal{A}) \rightarrow \operatorname{Sat}(\mathcal{A})$ in the category of partial orders, where $\times$ is the cartesian product. Items 2 and 3 in the previous definition make $\left(\operatorname{Sat}(\mathcal{A}),{ }^{\mathcal{A}}\right)$ a commutative semigroup in the category of partial orders.

Basic covers with operation provide a ground framework for studying the concepts we are mainly interested in, namely (commutative) quantales and locales. First, we shall show how to obtain presentations of (commutative) quantales. 


\section{Presenting commutative quantales: convergent covers}

From now on we assume $\mathcal{S}=(S, \triangleleft, \circ)$ to be a basic cover with operation (in the sense of definition 2.3). In this section we are going to study the case in which the corresponding structure $\left(\operatorname{Sat}(\mathcal{A}), \bigvee^{\mathcal{A}}, \circ^{\mathcal{A}}\right)$ is a commutative quantale (Mulvey [22] and Rosenthal [25]), that is, when multiplication $\circ^{\mathcal{A}}$ distributes over arbitrary joins. This we call a convergent cover. Together with a suitable notion of morphism, one gets a category which is dual to the category $\mathbf{C Q u}$ of commutative quantales.

Definition 3.1 A basic cover with operation $(S, \triangleleft, \circ)$ is called a convergent cover if ${ }^{\mathcal{A}}$ distributes over $\bigvee^{\mathcal{A}}$, that is, if $\left(\operatorname{Sat}(\mathcal{A}), \bigvee^{\mathcal{A}},{ }^{\mathcal{A}}\right)$ is a commutative quantale.

Our next aim is to obtain some more elementary characterizations of this notion.

Lemma 3.2 For every basic cover with operation $(S, \triangleleft, \circ)$, the following are equivalent:

(1) $\circ^{\mathcal{A}}$ distributes over $\bigvee^{\mathcal{A}}$, that is: $\bigvee_{i \in I}^{\mathcal{A}}\left(\mathcal{A} U_{i} \circ \mathcal{A} \mathcal{A} V\right)=\left(\bigvee_{i \in I}^{\mathcal{A}} \mathcal{A} U_{i}\right) \circ \mathcal{A} \mathcal{A} V$;

(2) $\circ$ distributes over $\bigcup$ modulo $=_{\mathcal{A}}$, that is: $\bigcup_{i \in I}\left(U_{i} \circ V\right)=_{\mathcal{A}}\left(\bigcup_{i \in I} U_{i}\right) \circ V$;

(3) $\circ$ is determined by its restriction on singletons, that is: $U \circ V={ }_{\mathcal{A}} \bigcup_{a \in U, b \in V}(\{a\} \circ$ $\{b\})$.

Proof By unfolding the definitions of $\circ^{\mathcal{A}}$ in (8) and $\bigvee^{\mathcal{A}}$ in (2) one sees that 2 is just a rewriting of 1 . Since $U=\bigcup_{a \in U}\{a\}$ and $V=\bigcup_{b \in V}\{b\}, 3$ follows by applying 2 twice. It remains to be checked that 3 implies $2: \bigcup_{i \in I}\left(U_{i} \circ V\right)=_{\mathcal{A}}$ (by 3) $\bigcup_{i \in I}\left(\bigcup_{a \in U_{i}, b \in V}\{a\} \circ\{b\}\right)=\bigcup_{i \in I, a \in U_{i}}\left(\bigcup_{b \in V}\{a\} \circ\{b\}\right)=\bigcup_{a \in \bigcup_{i \in I} U_{i}, b \in V}\{a\} \circ\{b\}=\mathcal{A}_{\mathcal{A}}$ (by 3 again) $\left(\bigcup_{i \in I} U_{i}\right) \circ V$.

From now on, we write $a \circ b$ for $\{a\} \circ\{b\}$ and more generally $a \circ V$ and $U \circ b$ for $\{a\} \circ V$ and $U \circ\{b\}$, respectively.

When $\circ$ is determined by its restriction on singletons (item 3 in the lemma), stability and localization become equivalent to their particular cases

$$
\frac{a \triangleleft U}{a \circ V \triangleleft U \circ V} \quad \frac{a \triangleleft U}{a \circ b \triangleleft U \circ b} \quad \frac{a \triangleleft U \quad b \triangleleft V}{a \circ b \triangleleft U \circ V}
$$

The equivalence between localization and the second rule in (10) will be crucial for inductive generation. In a similar way, associativity and commutativity become equivalent to $(a \circ b) \circ c \triangleleft a \circ(b \circ c)$ and to $a \circ b \triangleleft b \circ a$, respectively. So we have: 
Proposition 3.3 Let $(S, \triangleleft)$ be basic cover and let $\circ$ be an operation on $\mathcal{P}(S)$. Then $(S, \triangleleft, \circ)$ is a convergent cover if and only if all the following hold:

(1) $U \circ V={ }_{\mathcal{A}} \bigcup_{a \epsilon U, b \in V}(a \circ b)$

(2) $a \triangleleft U \& b \triangleleft V \Longrightarrow a \circ b \triangleleft U \circ V$ (or, equivalently: $a \triangleleft U \Longrightarrow a \circ b \triangleleft U \circ b$ )

(3) $(a \circ b) \circ c \triangleleft a \circ(b \circ c)$

(4) $a \circ b \triangleleft b \circ a$

(for all $a, b, c \in S$ and $U, V \subseteq S$ ).

We can characterize convergent covers also as basic covers with operation plus an extra operation defining implication, as follows. It is well known that a monotone function on a suplattice, which is an endofunctor on the corresponding poset category, preserves arbitrary joins if and only if it admits a right adjoint. This means that, given a basic cover with operation $(S, \triangleleft, \circ)$, the structure $\left(\operatorname{Sat}(\mathcal{A}), \bigvee^{A},{ }^{\mathcal{A}}\right)$ is a quantale, that is $\circ^{\mathcal{A}}$ distributes over $\bigvee^{\mathcal{A}}$, if and only if every map _-- $\circ^{\mathcal{A}} \mathcal{A} U$ has a right adjoint $\mathcal{A} U \rightarrow_{\mathcal{A} \text {--. }}$. In other words, a basic cover with operation $(S, \triangleleft, \circ)$ is a convergent cover iff there exists a binary operation $\rightarrow_{\mathcal{A}}$ on $\operatorname{Sat}(\mathcal{A})$ such that:

$$
\mathcal{A} W \circ^{\mathcal{A}} \mathcal{A} U \subseteq \mathcal{A} V \quad \Longleftrightarrow \mathcal{A} W \subseteq \mathcal{A} U \rightarrow \mathcal{A} \mathcal{A} V
$$

for all $U, V, W \subseteq S$. When it exists, $\rightarrow_{\mathcal{A}}$ satisfies:

$$
\mathcal{A} U \rightarrow_{\mathcal{A}} \mathcal{A} V=\bigvee^{\mathcal{A}}\left\{\mathcal{A} W \mid W \subseteq S \text { and } \mathcal{A} W \circ^{\mathcal{A}} \mathcal{A} U \subseteq \mathcal{A} V\right\} .
$$

By unfolding definitions, the right member becomes $\mathcal{A}(\bigcup\{W \subseteq S \mid W \circ U \triangleleft V\})$.

Now we give a predicative definition of $\rightarrow_{A}$. So assume $\rightarrow_{\mathcal{A}}$ to exist; thus $\circ^{\mathcal{A}}$ distributes over $\bigvee^{\mathcal{A}}$. By lemma 3.2, $\circ$ is determined by its restriction on singletons. So $W \circ U \triangleleft V$ becomes equivalent to $(\forall a \in W)(a \circ U \triangleleft V)$; hence $\mathcal{A} \bigcup\{W \subseteq S \mid$ $W \circ U \triangleleft V\}$ can be rewritten as $\mathcal{A}\{a \in S \mid a \circ U \triangleleft V\}$. Summing up, when $\rightarrow_{\mathcal{A}}$ exists, it is

$$
\mathcal{A} U \rightarrow_{\mathcal{A}} \mathcal{A} V=\mathcal{A}\{a \in S \mid a \circ U \triangleleft V\}
$$

(for all $U, V \subseteq S$ ). This suggests to define an operation

$$
U \rightarrow_{\mathcal{S}} V \stackrel{\text { def }}{=} \quad\{a \in S \mid a \circ U \triangleleft V\}
$$

on arbitrary subsets, for every basic cover with operation $\mathcal{S}=(S, \triangleleft, \circ)$, and then to put

$$
\mathcal{A} U \rightarrow_{\mathcal{A}} \mathcal{A} V \stackrel{\text { def }}{=} \mathcal{A}\left(U \rightarrow_{\mathcal{S}} V\right),
$$

which is an analogue of equation (8). Then one can show by localization that $\rightarrow_{\mathcal{A}}$ is well-defined on $\operatorname{Sat}(\mathcal{A})$. 
Proposition 3.4 For every basic cover with operation $\mathcal{S}=(S, \triangleleft, \circ)$, the following are equivalent:
a. $\mathcal{S}$ is a convergent cover
b. $\rightarrow_{\mathcal{A}}$ is right adjoint of $\circ^{\mathcal{A}}$
c. $\quad W \circ U \triangleleft V \Longleftrightarrow W \triangleleft U \rightarrow_{\mathcal{S}} V$ (for all $U, V, W \subseteq S$ ) with $\rightarrow_{\mathcal{S}}$ defined in (12).

Proof By the discussion above.

To represent unital commutative quantales [25], we also need the following:

Definition 3.5 We say that a convergent cover $(S, \triangleleft, \circ)$ is unital if there exists a subset $I \subseteq S$ such that $a \circ I={ }_{\mathcal{A}} a={ }_{\mathcal{A}} I \circ a$ for all $a \in S$.

\subsection{Morphisms between convergent covers}

Let $\mathcal{S}=\left(S, \triangleleft_{\mathcal{S}}, \circ_{\mathcal{S}}\right)$ and $\mathcal{T}=\left(T, \triangleleft_{\mathcal{T}}, \circ_{\mathcal{T}}\right)$ be two convergent covers. A morphism between the corresponding quantales $h: \operatorname{Sat}\left(\mathcal{A}_{\mathcal{T}}\right) \longrightarrow \operatorname{Sat}\left(\mathcal{A}_{\mathcal{S}}\right)$ is a map which preserves joins and multiplication. As in proposition 1.4, $h$ corresponds to a basic cover map $r$ from $\left(S, \triangleleft_{\mathcal{S}}\right)$ to $\left(T, \triangleleft_{\mathcal{T}}\right)$. Then the further condition on $h$ says that $r^{-}\left(U \circ_{\mathcal{T}} V\right)=\mathcal{A}_{\mathcal{S}} r^{-} U \circ_{\mathcal{S}} r^{-} V$. This equation is equivalent to its version on singletons since $r^{-}$is determined by its restriction on singletons. So we put:

Definition 3.6 Let $\mathcal{S}=\left(S, \triangleleft_{\mathcal{S}},{ }_{\mathcal{S}}\right)$ and $\mathcal{T}=\left(T, \triangleleft_{\mathcal{T}}, \circ_{\mathcal{T}}\right)$ be two convergent covers. A relation $r$ between $S$ and $T$ is a convergent cover map if:

- $\quad r$ is a basic cover map (that is, a morphism between basic covers);

- $r$ is convergent, that is

$$
r^{-}\left(b_{1} \circ_{\mathcal{T}} b_{2}\right) \quad=_{\mathcal{A}} \quad\left(r^{-} b_{1}\right) \circ_{\mathcal{S}}\left(r^{-} b_{2}\right)
$$

for all $b_{1}, b_{2} \in T$.

Two convergent cover maps are equal if they are equal as basic cover maps.

We then specialize the notion of convergent cover map in the presence of units:

Definition 3.7 Let $\mathcal{S}=\left(S, \triangleleft_{\mathcal{S}}, \circ_{\mathcal{S}}, I_{\mathcal{S}}\right)$ and $\mathcal{T}=\left(T, \triangleleft_{\mathcal{T}}, \circ_{\mathcal{T}}, I_{\mathcal{T}}\right)$ be two unital convergent covers. A relation $r$ between $S$ and $T$ is a unital convergent cover map if: 
- $\quad r$ is a convergent cover map;

- $r$ preserves the o-units, that is

$$
r^{-}\left(I_{\mathcal{T}}\right) \quad=_{\mathcal{A}} \quad I_{\mathcal{S}}
$$

Two unital convergent cover maps are equal if they are equal as basic cover maps.

Given the discussion above, it is straightforward to show that:

Proposition 3.8 Convergent covers with convergent cover maps form a subcategory of BCov, called CBCov. The category $\mathbf{C B C o v}$ is dual to the category $\mathbf{C Q u}$ of commutative quantales.

Proposition 3.9 Unital convergent covers with unital convergent cover maps form a subcategory of $\mathbf{C B C o v}$, called $\mathbf{u C B C o v}$. The category $\mathbf{u C B C o v}$ is dual to the category $\mathbf{u c Q u}$ of unital commutative quantales and commutative quantale maps preserving units.

The treatment of quantales in Battilotti and Sambin [1] is based on a binary operation $\bullet$ on the base $S$ of a basic cover. This operation can be seen as a very particular operation $\circ$ on subsets for which all $a \circ b$ are singletons. See section 5 below for further details.

A quite intuitive fact we shall need later is:

Lemma 3.10 Let $\mathcal{S}_{1}=\left(S, \triangleleft, \circ_{1}\right)$ and $\mathcal{S}_{2}=\left(S, \triangleleft, \circ_{2}\right)$ be two convergent covers (sharing the same underlying basic cover). If $\circ_{1}={ }_{\mathcal{A}} \circ_{2}$, that is $U \circ_{1} V={ }_{\mathcal{A}} U \circ_{2} V$ for all $U, V \subseteq S$, then $\mathcal{S}_{1}$ and $\mathcal{S}_{2}$ are isomorphic (in CBCov).

Proof The isomorphism is given by the identity relation on $S$.

\subsection{Inductive generation of convergent covers}

We now wish to extend the method of inductive generation from the case of basic covers to that of convergent covers. In order to generate a convergent cover (or, equivalently, a commutative quantale), it is quite natural to start from the following data: a set $S$ (that is, a set of generators of the corresponding suplattice); an axiom-set $I, C$ (encoding axioms of the form $a \triangleleft U$ ); a partial description of an operation on subsets given by its restriction to elements, namely a map $\delta: S \times S \longrightarrow \mathcal{P}(S)$ (we use a new symbol to underline the fact that, in the generation of a convergent cover, it is sufficient to define $\circ$ on singletons). 
The first step is to extend $\delta$ to an operation $\circ$ on $\mathcal{P}(S)$. This is simple: we put

$$
U \circ V=\bigcup_{a \in U, b \in V} \delta(a, b) .
$$

Recalling that $a \circ b$ stands for the subset $\{a\} \circ\{b\}$, one sees that $a \circ b=\delta(a, b)$ and hence $U \circ V=\bigcup_{a \in U, b \in V} a \circ b$, so that $\circ$ is determined by its restriction on singletons.

Next we add some conditions making the operation $\circ$ commutative and associative modulo $=_{\mathcal{A}}$. In order to apply the general scheme of inductive generation of basic covers, we are going to express such conditions as instances of the infinity rule. By using transitivity, one can see that commutativity is expressed by any one of the following equivalent conditions:

$$
b \circ c \triangleleft c \circ b \quad \frac{c \circ b \triangleleft U}{b \circ c \triangleleft U} \quad \frac{a \epsilon b \circ c \quad c \circ b \triangleleft U}{a \triangleleft U} .
$$

We choose the last one since it becomes an instance of the infinity rule provided that the axiom schema " $a \triangleleft c \circ b$ whenever $a \epsilon b \circ c$ " is encoded in the axiom-set. To this aim, it is sufficient first to enlarge the index set $I(a)$ by adding all pairs $(b, c)$ such that $a \epsilon b \circ c$ and then to define the corresponding cover of $a$ to be $c \circ b$.

Associativity is treated by following the same idea. One can see that

$(b \circ c) \circ d \triangleleft b \circ(c \circ d) \quad \frac{b \circ(c \circ d) \triangleleft U}{(b \circ c) \circ d \triangleleft U} \quad \frac{a \epsilon(b \circ c) \circ d \quad b \circ(c \circ d) \triangleleft U}{a \triangleleft U}$

are all equivalent. The last one becomes an instance of the infinity rule for a suitable extension of the axiom-set $I, C$.

We call $J, D$ the axiom-set extending $I, C$ in the way just described. By the above equivalences, the basic cover generated by $J, D$ is the least basic cover which makes $\circ$ commutative and associative modulo $=_{\mathcal{A}}$.

Thus it only remains to take care of localization. It is convenient to express it in the equivalent form given by the second rule in (10). In fact, as we now see, this allows us to show that localization can equivalently be expressed by a set-indexed family of conditions. A straightforward argument shows that the rules

$$
\frac{b \triangleleft V}{b \circ c \triangleleft V \circ c} \quad \frac{b \triangleleft V \quad V \circ c \triangleleft U}{b \circ c \triangleleft U} \quad \frac{a \epsilon b \circ c \quad b \triangleleft V \quad V \circ c \triangleleft U}{a \triangleleft U}
$$

are all equivalent. The last one looks more suitable for an inductive generation, since it resembles the infinity rule. However, it is not acceptable from a constructive point of view since the parameter $V$ ranges over a collection, namely $\mathcal{P}(S)$. Then the idea is 
to restrict the cover $V$ of $b$ to be one of those given by the axioms, namely $D(b, j)$ for $j \in J(b)$. This leads to the following rule:

$$
\frac{a \epsilon b \circ c \quad j \in J(b) \quad D(b, j) \circ c \triangleleft U}{a \triangleleft U} \quad \text { locax }
$$

(localization on axioms). This rule becomes an instance of the infinity rule for a suitable extension $J^{\prime}, D^{\prime}$ of the axiom-set $J, D$. In fact, it is sufficient to enlarge the index set $J(a)$ by adding triples $(b, j, c)$ such that $a \epsilon b \circ c$ and $j \in J(b)$ and then to define the corresponding cover of $a$ to be $D(b, j) \circ c$. The rule locax is equivalently expressed by

$$
\frac{j \in J(b) \quad D(b, j) \circ c \triangleleft U}{b \circ c \triangleleft U}
$$

which explains its name: every instance of the infinity rule for $J, D$ is "localized" to the basic neighbourhood $c$. Using the fact that $\circ$ is determined by its restriction on singletons, one can easily check that locax holds also for subsets, that is

$$
\frac{j \in J(b) \quad D(b, j) \circ V \triangleleft U}{b \circ V \triangleleft U} \quad \text { locax on subsets }
$$

for every $V \subseteq S$.

It is worth noting that the rule locax cannot be limited to the axioms of $I, C$. If that were the case, in fact, it would become impossible to prove the localized versions of commutativity and associativity. For instance, to prove $(a \circ b) \circ c \triangleleft(b \circ a) \circ c$ it is necessary to apply locax with respect to $J, D$ : for $x \in a \circ b$ one has $(a, b) \in J(x)$ and $D(x,(a, b))=b \circ a$ and hence from $(b \circ a) \circ c \triangleleft(b \circ a) \circ c$ by locax one gets $x \circ c \triangleleft(b \circ a) \circ c$. An alternative approach would be to consider locax for $I, C$ and then add commutativity and associativity already in localized form.

Summing up, starting from any axiom-set $I, C$ on a set $S$ and any map $\delta: S \times S \longrightarrow$ $\mathcal{P}(S)$, we first extended $\delta$ to a map $\circ: \mathcal{P}(S) \times \mathcal{P}(S) \longrightarrow \mathcal{P}(S)$ by putting $U \circ V=$ $\bigcup\{\delta(a, b) \mid a \in U, b \in V\}$ for all $U, V \subseteq S$. Then we called $J, D$ the axiom-set obtained from $I, C$ by adding axioms so that

$$
b \circ c \triangleleft c \circ b \quad \text { and } \quad(b \circ c) \circ d \triangleleft b \circ(c \circ d)
$$

become derivable. Finally, we let $J^{\prime}, D^{\prime}$ be the axiom-set extending $J, D$ with axioms making

$$
b \circ c \triangleleft D(b, j) \circ c
$$

derivable for all $b, c \in S$ and $j \in J(b)$. Then we can prove: 
Proposition 3.11 Let $I, C$ be an axiom-set on a set $S$ and let $\delta: S \times S \longrightarrow \mathcal{P}(S)$ be an arbitrary map. Define $\circ$ and $J^{\prime}, D^{\prime}$ as above and let $\triangleleft$ be the basic cover generated by $J^{\prime}, D^{\prime}$. Then $(S, \triangleleft, \circ)$ is a convergent cover in which $\triangleleft$ contains $\triangleleft_{I, C}$ (that is, $a \triangleleft_{I, C} U \Rightarrow a \triangleleft U$ for all $a \in S$ and $U \subseteq S$ ) and $\circ$ extends $\delta$ (that is, $a \circ b={ }_{\mathcal{A}} \delta(a, b)$ for all $a, b \in S$ ).

Moreover, if $\left(S, \triangleleft^{\prime}, \circ^{\prime}\right)$ is any convergent cover in which $\triangleleft^{\prime}$ contains $\triangleleft_{I, C}$ and $\circ^{\prime}$ extends $\delta$, then $\triangleleft^{\prime}$ contains $\triangleleft$ and $\circ^{\prime}=\mathcal{A}^{\prime} \circ$.

Proof The operation $\circ$ trivially extends $\delta$ and satisfies $U \circ V={ }_{\mathcal{A}} \bigcup_{a \in U, b \in V}(a \circ b)$. By the definition of $J^{\prime}, D^{\prime}$, the basic cover $\triangleleft$ contains $\triangleleft_{I, C}$ and associativity and commutativity hold. So, to show that $(S, \triangleleft, \circ)$ is a convergent cover, only localization remains to be proved. We prove $a \triangleleft U \Longrightarrow a \circ d \triangleleft U \circ d$ by induction on the proof of $a \triangleleft U$.

If $a \triangleleft U$ is obtained by reflexivity from $a \in U$, then by definition of $\circ$ we have $a \circ d$ $\subseteq U \circ d$ and hence $a \circ d \triangleleft U \circ d$ by reflexivity.

If $a \triangleleft U$ is obtained by infinity, we consider two cases according to whether the axiom used in the rule belongs to $J, D$ or not. In the former case, $a \triangleleft U$ is obtained from the assumptions: $j \in J(a)$ and $D(a, j) \triangleleft U$. By the inductive hypothesis applied to $D(a, j) \triangleleft U$, we get $D(a, j) \circ d \triangleleft U \circ d$ (pedantically, for each $b \in D(a, j)$, we use the inductive hypothesis $b \triangleleft U \Rightarrow b \circ d \triangleleft U \circ d$ ). This, together with $j \in J(a)$, implies $a \circ d \triangleleft U \circ d$ by locax on subsets (that is, by a suitable instance of the infinity rule for $\left.J^{\prime}, D^{\prime}\right)$.

We now analyze the case in which $a \triangleleft U$ is obtained by infinity from an axiom of $J^{\prime}, D^{\prime}$ that does not belong to $J, D$. In other words, $a \triangleleft U$ is derived from the assumptions $a \epsilon b \circ c, j \in J(b)$ and $D(b, j) \circ c \triangleleft U$ and hence the infinity rule corresponding to this case is precisely locax. The inductive hypothesis on the assumption $D(b, j) \circ c \triangleleft U$ gives $(D(b, j) \circ c) \circ d \triangleleft U \circ d$ and hence $D(b, j) \circ(c \circ d) \triangleleft U \circ d$ by associativity. This, together with $j \in J(b)$, yields $b \circ(c \circ d) \triangleleft U \circ d$ by locax on subsets and hence $(b \circ c) \circ d \triangleleft U \circ d$ by associativity again. So $a \circ d \triangleleft U \circ d$ as wished, because $a \epsilon b \circ c$ and $\circ$ is determined by its restriction on singletons.

Finally, let $\left(S, \triangleleft^{\prime}, \circ^{\prime}\right)$ be a convergent cover in which $\triangleleft^{\prime}$ contains $\triangleleft_{I, C}$ and such that $a \circ^{\prime} b=\mathcal{A}^{\prime} \delta(a, b)$. Then $U \circ^{\prime} V={ }_{\mathcal{A}^{\prime}} \bigcup_{a \epsilon U, b \in V} \delta(a, b)=U \circ V$. So also $\left(S, \triangleleft^{\prime}, \circ\right)$ is a convergent cover (in fact, it is isomorphic to $\left(S, \triangleleft^{\prime}, \circ^{\prime}\right)$ by lemma 3.10) and hence $\triangleleft^{\prime}$ fulfills all the axioms in $J^{\prime}, D^{\prime}$. So $\triangleleft^{\prime}$ contains $\triangleleft$.

Remark 3.12 In order to generate a unital convergent cover, it is sufficient to start with an additional piece of data, namely a subset $I$, and then impose extra axioms 
about unit $a \triangleleft a \circ I$ and $a \circ I \triangleleft a$. Note that, in the presence of commutativity and associativity, it is irrelevant whether the unit axioms added before or after localizing the axioms.

Definition 3.13 A convergent cover $(S, \triangleleft, \circ)$ is called inductively generated if it constructed as in proposition 3.11 for some axiom-set $I, C$ over $S$ and some map $\delta: S \times S \rightarrow P(S)$.

We call $\mathbf{C B C o v}_{i}$ the full subcategory of $\mathbf{C B C o v}$ whose objects are inductively generated. Similarly, $\mathbf{u C B C o v}{ }_{i}$ is the full subcategory of inductively generated objects in uCBCov.

We end with a lemma characterizing convergent cover maps between inductively generated unital convergent covers:

Lemma 3.14 Let $\mathcal{S}=\left(S, \triangleleft_{\mathcal{S}}, \circ_{\mathcal{S}}, I_{\mathcal{S}}\right)$ and $\mathcal{T}=\left(T, \triangleleft \mathcal{T}, \circ_{\mathcal{T}}, I_{\mathcal{T}}\right)$ be two unital convergent covers. Assume that $\mathcal{T}$ is inductively generated by means of an axiom-set $I, C$ and a map $\delta: T \times T \longrightarrow \mathcal{P}(T)$ according to proposition 3.11. Then a relation $r$ between $S$ and $T$ is a unital convergent cover map from $\mathcal{S}$ to $\mathcal{T}$ if and only if the following hold:

(1) $r^{-} a \triangleleft \mathcal{S} r^{-} C(a, i)$ for all $a \in T$ and all $i \in I(a)$;

(2) $r^{-} \delta(a, b)={ }_{\mathcal{A}} r^{-} a \circ_{\mathcal{S}} r^{-} b$ for all $a, b \in T$;

(3) $r^{-} I_{\mathcal{T}}={ }_{\mathcal{A}} I_{\mathcal{S}}$.

Proof By the definition of $\circ_{\mathcal{T}}$ (in proposition 3.11), item 2 is equivalent to $r^{-}(a \circ \mathcal{T} b)$ $=_{\mathcal{A}}\left(r^{-} a\right) \circ_{\mathcal{S}}\left(r^{-} b\right)$. So we must check only that condition 1 is tantamount to $r$ being a basic cover map. By lemma 1.6, it is sufficient to show that 1 holds also for the other axioms of $J^{\prime}, D^{\prime}$, namely commutativity, associativity and localization. In fact, condition 1 for these extra axioms follows from 2 and the corresponding properties of $\mathcal{S}$. We check this only in two cases.

First, we see that $r^{-} a \triangleleft_{\mathcal{S}} r^{-}(c \circ \mathcal{T} b)$ whenever $a \epsilon b \circ \mathcal{T} c$, namely that condition 1 for one of the commutativity axioms of $J, D$. By 2 and commutativity of $\circ_{\mathcal{S}}$ we have $r^{-}(c \circ \mathcal{T} b)=_{\mathcal{A}}\left(r^{-} c\right) \circ_{\mathcal{S}}\left(r^{-} b\right)=_{\mathcal{A}}\left(r^{-} b\right) \circ_{\mathcal{S}}\left(r^{-} c\right)=_{\mathcal{A}} r^{-}(b \circ \mathcal{T} c)$. So the claim is equivalent to $r^{-} a \triangleleft r^{-}(b \circ \mathcal{T} c)$. But this follows from $r^{-} a \subseteq r^{-}(b \circ \mathcal{T} c)$ which in turn is a consequence of the assumption $a \epsilon b \circ \mathcal{T} c$.

Second, we consider a particular instance of locax. Assume $a \epsilon b \circ \mathcal{T} c$ and $C(b, i) \circ_{\mathcal{T}}$ $c \triangleleft_{\mathcal{T}} U$ for some $b, c \in T$ and some $i \in I(b)$. Then $r^{-}\left(C(b, i) \circ_{\mathcal{T}} c\right) \triangleleft_{\mathcal{S}} r^{-} U$ 
by inductive hypothesis and hence $\left(r^{-} C(b, i)\right) \circ_{\mathcal{S}}\left(r^{-} c\right) \triangleleft_{\mathcal{S}} r^{-} U$ by 2 . From 1 by localization in $\mathcal{S}$ one can deduce $\left(r^{-} b\right) \circ_{\mathcal{S}}\left(r^{-} c\right) \triangleleft_{\mathcal{S}}\left(r^{-} C(b, i)\right) \circ_{\mathcal{S}}\left(r^{-} c\right)$. So by transitivity $\left(r^{-} b\right) \circ_{\mathcal{S}}\left(r^{-} c\right) \triangleleft_{\mathcal{S}} r^{-} U$ and hence $r^{-}(b \circ \mathcal{T} c) \triangleleft_{\mathcal{S}} r^{-} U$ by 2. Since $a \epsilon b \circ \mathcal{T} c$ by assumption, one can conclude $r^{-} a \triangleleft_{\mathcal{S}} r^{-} U$.

\subsection{Categorical reading of convergence}

In this section we wish to show that the category $\mathbf{C B C o v}_{i}$ is equivalent to the category of commutative co-semigroups in $\mathbf{B C o v}_{i}$. This result expresses in a constructive way the fact that $\mathbf{C Q u}$ (commutative quantales) is equivalent to the category of commutative semigroups in SupLat (see Joyal and Tierney [13]). Once this is achieved, it is straightforward to obtain a description of commutative co-monoids in $\mathbf{B C o v} i$ simply by considering unital convergent covers. Similarly, one can easily extends these results to the non-commutative case.

Definition 3.15 A commutative co-semigroup in the category $\mathbf{B C o v}_{i}$ is an inductively generated basic cover $\mathcal{S}$ together with a map $\mu: \mathcal{S} \rightarrow \mathcal{S} \otimes \mathcal{S}$ in $\mathbf{B C o v}_{i}$ such that the following diagrams commute.
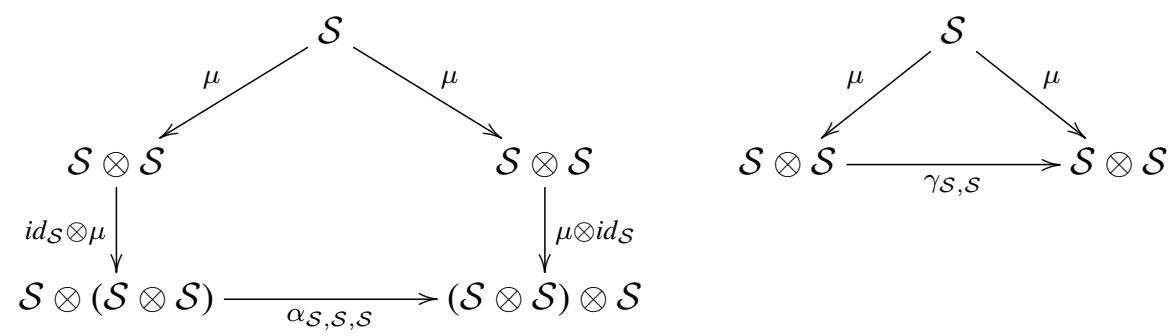

A morphism between two commutative co-semigroups $(\mathcal{S}, \mu)$ and $\left(\mathcal{S}^{\prime}, \mu^{\prime}\right)$ is a basic cover map $r: \mathcal{S} \longrightarrow \mathcal{S}^{\prime}$ such that the following diagram commutes.

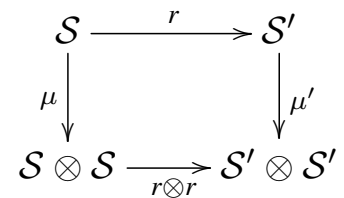

Lemma 3.16 Every inductively generated convergent cover can be seen as a commutative co-semigroup in $\mathbf{B C o v}_{i}$.

Conversely every commutative co-semigroup in $\mathbf{B C o v}_{i}$ determines a convergent basic cover. 
Proof Given a convergent cover $\mathcal{S}=(S, \triangleleft, \circ)$, let us define

$$
\mu_{\circ}: \mathcal{S} \longrightarrow \mathcal{S} \otimes \mathcal{S} \quad \text { as } \quad c \mu_{\circ}(a, b) \equiv c \epsilon a \circ b
$$

for all $c, a, b \in S$. That is, we put $\mu_{\circ}{ }^{-}(a, b)=a \circ b$. In order to prove that $\mu_{\circ}$ is a basic cover map, by lemma 1.6 it is sufficient to check that $\mu_{\circ}{ }^{-}(a, b) \triangleleft \mu_{\circ}{ }^{-} E((a, b), j)$ holds for every axiom $(a, b) \triangleleft E((a, b), j)$ of $\mathcal{S} \otimes \mathcal{S}$. By symmetry, we can consider only the case $E((a, b), j)=C(a, i) \times\{b\}$ for some axiom $a \triangleleft C(a, i)$ of $\mathcal{S}$. From $a \triangleleft C(a, i)$ by localization one has $a \circ b \triangleleft C(a, i) \circ b$ and so:

$$
\mu_{\circ}{ }^{-}(a, b)=a \circ b \triangleleft C(a, i) \circ b={ }_{\mathcal{A}} \bigcup_{c \in C(a, i)} c \circ b .
$$

Since $c \circ b=\mu_{\circ}{ }^{-}(c, b)$ and since $\mu_{\circ}{ }^{-}$distributes over unions, one gets

$$
\bigcup_{c \in C(a, i)} c \circ b=\mu_{\circ}{ }^{-}\left(\bigcup_{c \in C(a, i)}\{(c, b)\}\right)=\mu_{\circ}^{-}(C(a, i) \times\{b\}) .
$$

So we can conclude that $\mu_{\circ}{ }^{-}(a, b) \triangleleft \mu_{\circ}{ }^{-} E((a, b), j)$.

Commutativity and associativity of $\left((S, \triangleleft), \mu_{\circ}\right)$ follow from the fact that $\circ$ is commutative and associative modulo $=_{\mathcal{A}}$. For instance, the equality $\gamma_{\mathcal{S}, \mathcal{S}} \cdot \mu_{\circ}={ }_{\mathbf{B C o v}} \mu_{\circ}$ means that $\mu_{\circ}{ }^{-} \gamma_{\mathcal{S}, \mathcal{S}}{ }^{-}(a, b)={ }_{\mathcal{A}} \mu_{\circ}{ }^{-}(a, b)$ for all $(a, b) \in S \times S$; by unfolding definitions and since $\mu_{\circ}$ respects covers, this becomes $\mu_{\circ}{ }^{-}(b, a)={ }_{\mathcal{A}} a \circ b$, that is, $b \circ a={ }_{\mathcal{A}} a \circ b$.

Conversely, given a commutative co-semigroup with $\mu: \mathcal{S} \longrightarrow \mathcal{S} \otimes \mathcal{S}$, we put

$$
U \circ_{\mu} V=\mathcal{A} \mu^{-}(U \times V)
$$

for all $U, V \subseteq S$. Note that this definition respects the equality of morphisms (definition $1.3)$.

By (7), $\mathcal{A} U \times \mathcal{A} V={ }_{\mathcal{A}_{\mathcal{S} \otimes \mathcal{S}}} U \times V$ holds and hence $\mu^{-}(\mathcal{A} U \times \mathcal{A} V)={ }_{\mathcal{A}} \mu^{-}(U \times V)$ because $\mu$ respects covers. By the definition of $\circ_{\mu}$, this means that $\mathcal{A} U \circ_{\mu} \mathcal{A V}={ }_{\mathcal{A}}$ $U \circ_{\mu} V$, so that $\circ_{\mu}$ is well-defined (proposition 2.1). The operation $\circ_{\mu}$ is determined by its restriction on singletons:

$$
U \circ_{\mu} V={ }_{\mathcal{A}} \mu^{-}(U \times V)=\bigcup_{u \in U, v \in V} \mu^{-}(u, v)=\mathcal{A}_{\mathcal{A}} \bigcup_{u \in U, v \in V} u \circ_{\mu} v .
$$

Finally, $\circ_{\mu}$ is associative and commutative modulo $=_{\mathcal{A}}$ because so is the co-semigroup. For instance, by the equation $\alpha_{\mathcal{S}, \mathcal{S}, \mathcal{S}} \cdot\left(\operatorname{id}_{\mathcal{S}} \otimes \mu\right) \cdot \mu=\left(\mu \otimes \mathrm{id}_{\mathcal{S}}\right) \cdot \mu$ one has

$$
\mu^{-}\left(\operatorname{id}_{\mathcal{S}} \otimes \mu\right)^{-} \alpha_{\mathcal{S}, \mathcal{S}, \mathcal{S}}((U \times V) \times W) \quad={ }_{\mathcal{A}} \quad \mu^{-}\left(\mu \otimes \operatorname{id}_{\mathcal{S}}\right)^{-}((U \times V) \times W)
$$

for all $U, V, W \subseteq S$. By the definition of $\alpha_{\mathcal{S}, \mathcal{S}, \mathcal{S}}$ and of the tensor of two morphisms, this gives $\left.\mu^{-}\left(U \times \mu^{-}(V \times W)\right)={ }_{\mathcal{A}} \mu^{-}\left(\mu^{-}(U \times V) \times W\right)\right)$. By the definition of $\circ_{\mu}$ this is precisely $U \circ_{\mu}\left(V \circ_{\mu} W\right)=_{\mathcal{A}}\left(U \circ_{\mu} V\right) \circ_{\mu} W$. 
Proposition 3.17 The category $\mathbf{C B C o v}_{i}$ of inductively generated convergent covers is equivalent to the category of commutative co-semigroups in $\mathbf{B C o v}_{i}$.

Proof Thanks to the previous lemma, to each object $(S, \triangleleft, \circ)$ in $\mathbf{C B C o v}_{i}$ we associate the commutative co-semigroup $\left((S, \triangleleft), \mu_{\circ}\right)$. Conversely, to every commutative co-semigroup $((S, \triangleleft), \mu)$ we associate $\left(S, \triangleleft, \circ_{\mu}\right)$. By lemma 3.10, $(S, \triangleleft, \circ)$ is isomorphic to $\left(S, \triangleleft, \circ_{\mu_{\circ}}\right)$ because $U \circ_{\mu_{\circ}} V=\mathcal{A} \mu_{\circ}{ }^{-}(U \times V)=\mathcal{A} \bigcup_{a \epsilon U, b \in V} \mu_{\circ}{ }^{-}(a, b)$ $=\mathcal{A} \bigcup_{a \epsilon U, b \in V} a \circ b=\mathcal{A}(U \circ V)$. Moreover, $((S, \triangleleft), \mu)$ coincides with $\left((S, \triangleleft), \mu_{\circ_{\mu}}\right)$ because $\mu_{\circ_{\mu}}{ }^{-}(a, b)=a \circ_{\mu} b=\mathcal{A} \mu^{-}(a, b)$, for all $a, b \in S$ and so $\mu_{\circ_{\mu}}={ }_{\mathbf{B C o v}_{i}} \mu$.

Morphisms between co-semigroups correspond to convergent cover maps. In fact, a basic cover map $r: \mathcal{S} \longrightarrow \mathcal{S}^{\prime}$ is a co-semigroup map from $(\mathcal{S}, \mu)$ to $\left(\mathcal{S}^{\prime}, \mu^{\prime}\right)$ if and only if $\mu^{\prime} \cdot r={ }_{\mathbf{B C o v}_{i}}(r \otimes r) \cdot \mu$ iff $r^{-} \mu_{\mathcal{S}^{\prime}}{ }^{-}(a, b)={ }_{\mathcal{A}} \mu_{\mathcal{S}}{ }^{-}(r \otimes r)^{-}(a, b)$ for all $a, b \in S^{\prime}$. This means precisely that $r^{-}\left(a \circ_{\mu^{\prime}} b\right)={ }_{\mathcal{A}} r^{-} a \circ_{\mu} r^{-} b$, that is $r$ is a convergent cover map from $\left(S, \triangleleft, \circ_{\mu}\right)$ to $\left(S^{\prime}, \triangleleft^{\prime}, \circ_{\mu^{\prime}}\right)$. Vice versa, $r:(S, \triangleleft, \circ) \longrightarrow\left(S^{\prime}, \triangleleft^{\prime}, \circ^{\prime}\right)$ is a convergent cover map iff $r^{-}\left(a \circ^{\prime} b\right)={ }_{\mathcal{A}} r^{-} a \circ r^{-} b$, that is, $r^{-} \mu_{\circ^{\prime}}-(a, b)={ }_{\mathcal{A}} \mu_{\circ}{ }^{-}(r \otimes r)^{-}(a, b)$. This means that $\mu_{\circ} \cdot r={ }_{\mathbf{B C o v}}(r \otimes r) \cdot \mu_{\circ}$, that is $r$ is a morphism of co-semigroups from $\left((S, \triangleleft), \mu_{\circ}\right)$ to $\left(\left(S^{\prime}, \triangleleft^{\prime}\right), \mu_{\circ^{\prime}}\right)$.

The restriction to inductive generated structures in the previous statement is needed only to be sure that $\otimes$ exists predicatively. In an impredicative framework, the above result states that the whole category $\mathbf{C B C o v}$ is equivalent to the category of commutative cosemigroups in BCov. By passing to the opposite categories, one gets that (commutative) quantales are equivalent to (commutative) semigroups over suplattices.

Definition 3.18 A commutative co-monoid in the category $\mathbf{B C o v}_{i}$ is a commutative co-semigroup $(\mathcal{S}, \mu)$ in $\mathbf{B C o v}_{i}$ together with a map $\eta: \mathcal{S} \rightarrow E$ such that the following diagram commutes.

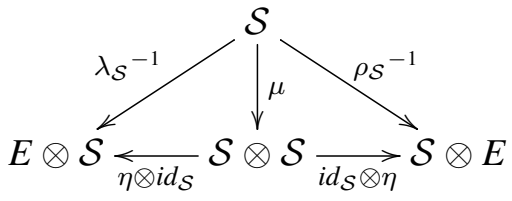

Analogously to the previous result, one can show that:

Corollary 3.19 The category $\mathbf{u C B C o v}_{i}$ of inductively generated unital convergent covers is equivalent to the category of commutative co-monoids in $\mathbf{B C o v}_{i}$. 


\section{Predicative locales: formal covers}

In this section we finally come to the case of locales. It is common to associate the name "Formal Topology" with a predicative version of the theory of Locales. However, following its first appearance in Sambin [26], we prefer to conceive formal topologies as a predicative version of open (also known as overt) locales (see Joyal and Tierney [13]). Predicative presentations of locales tout court are called here formal covers.

As usual a locale or frame is a suplattice in which binary meets distribute over arbitrary joins. It is well known that a quantale is a locale exactly when its multiplication coincides with the order-theoretic meet. In our framework, the quantale $\left(\operatorname{Sat}(\mathcal{A}), \bigvee^{\mathcal{A}}, \circ^{\mathcal{A}}\right)$ presented by a convergent cover $(S, \triangleleft, \circ)$ is actually a locale when

$$
\mathcal{A} U \circ^{\mathcal{A}} \mathcal{A} V=\mathcal{A} U \wedge^{\mathcal{A}} \mathcal{A} V
$$

for every $U, V \subseteq S$. By unfolding definitions, this reduces to $\mathcal{A}(U \circ V)=\mathcal{A} U \cap \mathcal{A} V$ for every $U, V \subseteq S$.

Definition 4.1 A convergent cover $(S, \triangleleft, \circ)$ is called a formal cover if $\circ \mathcal{A}=\wedge^{\mathcal{A}}$, that is:

$$
\mathcal{A}(U \circ V)=\mathcal{A} U \cap \mathcal{A} V
$$

for all $U, V \subseteq S$. In this case, $\left(\operatorname{Sat}(\mathcal{A}), \bigvee^{\mathcal{A}}, \circ^{\mathcal{A}}\right)$ is a locale.

Lemma 4.2 For every convergent cover all the items in each column are equivalent one to another:

A1. $\mathcal{A}(U \circ V) \subseteq \mathcal{A} U \cap \mathcal{A} V$

A2. $\quad U \circ V \triangleleft U$ and $U \circ V \triangleleft V$ (weakening)

A3. $\quad \frac{U \triangleleft W}{U \circ V \triangleleft W}$ and $\frac{V \triangleleft W}{U \circ V \triangleleft W} \quad$ (o-left)

A4. $\quad a \circ b \triangleleft a$ and $a \circ b \triangleleft b \quad$ (weakening)

A5. $\quad \frac{a \triangleleft W}{a \circ b \triangleleft W}$ and $\frac{b \triangleleft W}{a \circ b \triangleleft W} \quad$ (o-left)
B1. $\mathcal{A} U \cap \mathcal{A} V \subseteq \mathcal{A}(U \circ V)$

B2. $U \triangleleft U \circ U$ (contraction)

B3. $\frac{W \triangleleft U \quad W \triangleleft V}{W \triangleleft U \circ V}$ (o-right)

B4. $a \triangleleft a \circ a \quad$ (contraction)

B5. $\frac{a \triangleleft U \quad a \triangleleft V}{a \triangleleft U \circ V} \quad$ (o-right)

Summing up, the following are equivalent:

i) $\circ^{\mathcal{A}}=\wedge^{\mathcal{A}}$, that is $\mathcal{A}(U \circ V)=\mathcal{A} U \cap \mathcal{A} V$; 
ii) weakening and contraction axioms;

iii) o-left and o-right.

Proof $A 2$ is just a rewriting of $A 1$ and it is also a special case of $A 3$, which in turn is obtained from $A 2$ by transitivity. $A 4$ is a special case of $A 2$. Conversely, since $U \circ V$ is determined by its restriction to singletons, it is sufficient to show that $a \circ b \triangleleft U$ and $a \circ b \triangleleft V$ for all $a \in U$ and $b \in V$. These follow immediately from $A 4$ by reflexivity and transitivity. Finally, $A 4$ is a special case of $A 5$ and the converse holds by transitivity.

$B 1$ is a rewriting of $B 5 . B 4$ is a special case of $B 5$. Conversely, from the premises of $B 5$ one has $a \circ a \triangleleft U \circ V$ by stability and hence $a \triangleleft U \circ V$ by $B 4$ and transitivity. The equivalence between $B 2$ and $B 3$ is proved similarly. Finally, $B 3$ is clearly equivalent to $B 5$.

This leads to a number of characterizations of formal covers. For instance, formal covers are precisely the convergent covers satisfying weakening and contraction. We have also the following.

Proposition 4.3 A structure $\mathcal{S}=(S, \triangleleft, \circ)$ is a formal cover if and only if $(S, \triangleleft)$ is a basic cover and $\circ$ is a binary operation on subsets of $S$ such that:

(1) $U \circ V={ }_{\mathcal{A}} \bigcup_{a \in U, b \in V}(a \circ b) \quad$ ( $\circ$ is determined by its restriction on singletons)

(2) $a \triangleleft U \& a \triangleleft V \Longrightarrow a \triangleleft U \circ V \quad$ (०-right)

(3) $a \circ b \triangleleft a$ and $a \circ b \triangleleft b$

(weakening)

for all $a, b, c \in S$ and $U, V \subseteq S$.

Proof Stability, associativity and commutativity are all derivable from $\circ$-right and weakening. In fact, if $a \triangleleft U$ and $b \triangleleft V$, then $a \circ b \triangleleft U$ and $a \circ b \triangleleft V$ by weakening (and transitivity); so $a \circ b \triangleleft U \circ V$ by $\circ$-right. This proves stability. Commutativity is easy: since $a \circ b$ is covered both by $b$ and $a$ (weakening), it is also covered by $b \circ a$ ( $\circ$-right). Finally, we check associativity in the form $(a \circ b) \circ c \triangleleft a \circ(b \circ c)$. By $\circ$-right it is sufficient to prove $(a \circ b) \circ c \triangleleft a$ and $(a \circ b) \circ c \triangleleft b \circ c$. The former follows by combining ( $a \circ b) \circ c \triangleleft a \circ b$ and $a \circ b \triangleleft a$ which both hold by weakening. To prove the latter it is sufficient to have both $(a \circ b) \circ c \triangleleft b$ and $(a \circ b) \circ c \triangleleft c$ which, again, follow by weakening and transitivity.

Note that every formal cover is unital: 
Proposition 4.4 If $\mathcal{S}=(S, \triangleleft, \circ)$ is a formal cover, then $S$ is a unit for $\circ$.

Proof $a \circ S={ }_{\mathcal{A}} a$ holds thanks to weakening and contraction.

This proposition says that, impredicatively, every frame is a unital quantale.

\subsection{Morphisms between formal covers}

Morphisms between frames are functions preserving both arbitrary joins and finite meets. The category of frames is called Frm. The category of locales Loc is usually introduced as the opposite of Frm. So objects are the same, while morphisms of locales are usually just morphisms of frames "in the opposite direction". Contrary to this common practice, one can provide an intuitive topological definition of morphisms between formal covers (see Maietti and Valentini [20] and Sambin [30]).

When frames are seen as particular unital quantales, their morphisms are precisely unital quantale morphisms. In our framework, thanks to proposition 4.4, we put:

Definition 4.5 The full subcategory of $\mathbf{u C B C o v}$ whose objects are formal covers is called FCov. A unital convergent cover map between two formal covers is called a formal cover map.

In particular, a formal cover map $r: \mathcal{S} \rightarrow \mathcal{T}$ is total, that is, it satisfies the equation $r^{-} T=\mathcal{A}_{\mathcal{S}} S$.

Proposition 4.6 FCov is impredicatively dual to Frm and equivalent to Loc.

Proof Recall that $\mathbf{u C B C o v}$ is dual to $\mathbf{u c Q u}$ (proposition 3.9). So it is sufficient to observe that a convergent cover is a formal cover iff the corresponding quantale is a locale.

If $\mathcal{S}=(S, \triangleleft, \circ)$ and $\mathcal{S}^{\prime}=\left(S, \triangleleft, \circ^{\prime}\right)$ are two formal covers with the same underlying basic cover, then $U \circ V={ }_{\mathcal{A}} U \circ^{\prime} V$ for all $U, V \subseteq S$ by the definition of formal cover. So $\mathcal{S}$ and $\mathcal{S}^{\prime}$ are isomorphic as convergent covers by lemma 3.10. Since the isomorphism provided by that lemma is given by the identity relation on $S$, it is also total and hence it is an isomorphism of formal covers. This proves the following: 
Lemma 4.7 Two formal covers sharing the same underlying basic cover are isomorphic. In other words, given a basic cover $(S, \triangleleft)$, there exists, up to isomorphism of formal covers, at most one operation $\circ$ such that $(S, \triangleleft, \circ)$ is a formal cover.

We will see (lemma 5.4) that, when it exists, the operation $\circ$ of a formal cover coincides up to $=_{\mathcal{A}}$ with an operation that can be characterized explicitly in terms of the cover (see (18) below). Hence, while for convergent covers the operation $\circ$ is new structure, for a formal cover the existence of $\circ$ becomes a property.

\subsection{Inductive generation of formal covers}

In this section we extend the method for generating basic covers (suplattices) and convergent covers (commutative quantales) to the case of formal covers (locales). Given an axiom-set $I, C$ and a map $\delta$ as in proposition 3.11, we consider the axiom-set $J^{\prime}, D^{\prime}$ constructed there. Here we define a further axiom-set, say $J^{\prime \prime}, D^{\prime \prime}$, by enlarging $J^{\prime}, D^{\prime}$ in a suitable way in order to take care of the extra axiom schemata $a \circ b \triangleleft a$ (weakening) and $a \triangleleft a \circ a$ (contraction).

Definition 4.8 Let $I, C$ be an axiom-set on a set $S$ and let $\delta: S \times S \longrightarrow \mathcal{P}(S)$ be an arbitrary map. We put $U \circ V=\bigcup\{\delta(a, b) \mid a \in U, b \in V\}$. We call $J^{\prime \prime}, D^{\prime \prime}$ the axiom-set obtained by enlarging the axiom-set $J^{\prime}, D^{\prime}$ in section 3.2 with the axioms of the form

$$
b \circ c \triangleleft c \quad a \triangleleft a \circ a
$$

for $a, b, c$ in $S$. Formally, for every $a \in S, J^{\prime \prime}(a)$ is obtained from $J^{\prime}(a)$ by adding a new index for every pair $(b, c)$ such that $a \epsilon b \circ c$ and a single further index $*$. Then one adds respectively the axioms $a \triangleleft c$ for every $(b, c)$ and $a \triangleleft a \circ a$.

Proposition 4.9 Let $I, C$ be an axiom-set on a set $S$ and let $\delta: S \times S \longrightarrow \mathcal{P}(S)$ be an arbitrary map. We put $U \circ V=\bigcup\{\delta(a, b) \mid a \epsilon U, b \in V\}$ and we consider the basic cover $\triangleleft$ generated by the axiom-set $J^{\prime \prime}, D^{\prime \prime}$ described above. Then $(S, \triangleleft, \circ)$ is the least formal cover containing $\triangleleft_{I, C}$ (that is, $a \triangleleft_{I, C} U \Rightarrow a \triangleleft U$ for all $a \in S$ and $U \subseteq S$ ) and extending $\delta$ (that is, $a \circ b={ }_{\mathcal{A}} \delta(a, b)$ for all $a, b \in S$ ).

Proof The claim is almost obvious after proposition 3.11. One should only note that it is not necessary to modify locax in order to take care of the new axioms of weakening and contraction. For instance, the localized form of weakening, namely $(a \circ b) \circ c \triangleleft a \circ c$, follows already from its standard form. In fact, by associativity and commutativity, it is equivalent to $(a \circ c) \circ b \triangleleft a \circ c$ which holds by weakening. 
Definition 4.10 A formal cover $(S, \triangleleft, \circ)$ is inductively generated if it is constructed as in proposition 4.9 for some axiom-set $I, C$ over $S$ and some map $\delta: S \times S \longrightarrow$ $P(S)$.

We call $\mathbf{F C o v}$ the full subcategory of $\mathbf{F C o v}$ whose objects are inductively generated.

It is straightforward to extend lemma 3.14 to the framework of formal covers.

Lemma 4.11 Let $\mathcal{S}=\left(S, \triangleleft_{\mathcal{S}},{ }_{\mathcal{S}}\right)$ and $\mathcal{T}=\left(T, \triangleleft_{\mathcal{T}}, \circ_{\mathcal{T}}\right)$ be two formal covers. Assume that $\mathcal{T}$ is inductively generated by means of an axiom-set $I, C$ and a map $\delta: T \times T$ $\longrightarrow \mathcal{P}(T)$ according to proposition 4.9. Finally, let $r$ be a relation between $S$ and $T$. Then $r$ is a formal cover map from $\mathcal{S}$ to $\mathcal{T}$ if and only if the following hold:

(1) $r^{-} a \triangleleft r^{-} C(a, i)$ for all $a \in T$ and all $i \in I(a)$;

(2) $r^{-} \delta(a, b)={ }_{\mathcal{A}}\left(r^{-} a\right) \circ\left(r^{-} b\right)$ for all $a, b \in T$;

(3) $r^{-} T=\mathcal{A} S$.

The following picture summarizes the main definitions of this paper.

\begin{tabular}{|c|c|c|}
\hline & $\begin{array}{c}\text { STRUCTURE } \\
\text { ON OPENS }\end{array}$ & MORPHISMS \\
\hline $\begin{array}{c}\text { basic cover } \\
\mathcal{S}=(S, \triangleleft)\end{array}$ & $\begin{array}{c}\text { suplattice } \\
\left(\operatorname{Sat}(\mathcal{A}), \bigvee^{\mathcal{A}}\right)\end{array}$ & $\begin{array}{c}\text { basic cover map }= \\
r: S \rightarrow T \text { relation }+ \\
b \triangleleft_{\mathcal{T}} V \Rightarrow r^{-} b \triangleleft_{\mathcal{S}} r^{-} V\end{array}$ \\
\hline $\begin{array}{c}\text { convergent cover } \\
\mathcal{S}=(S, \triangleleft, \circ) \\
\text { basic cover }+ \text { associativity }+ \\
\text { commutativity }+ \text { localization }+ \\
\text { o determined by singletons }\end{array}$ & $\begin{array}{l}\text { commutative quantale } \\
\quad\left(\operatorname{Sat}(\mathcal{A}), \bigvee^{\mathcal{A}}, \circ^{\mathcal{A}}\right)\end{array}$ & $\begin{array}{c}\text { convergent cover map }= \\
\text { basic cover map }+ \\
r^{-}\left(a \circ_{\mathcal{T}} b\right)={ }_{\mathcal{A}} r^{-} a \circ_{\mathcal{S}} r^{-} b\end{array}$ \\
\hline $\begin{array}{c}\text { unital convergent cover } \\
\qquad \mathcal{S}=(S, \triangleleft, \circ, I) \\
\text { convergent cover }+a={ }_{\mathcal{A}} a \circ I \\
\end{array}$ & $\begin{array}{l}\text { unital comm. quantale } \\
\left(\operatorname{Sat}(\mathcal{A}), \bigvee^{\mathcal{A}},{ }^{\mathcal{A}}, \mathcal{A} I\right)\end{array}$ & $\begin{array}{c}\text { unital conv. cover map = } \\
\text { convergent cover map }+ \\
r^{-} I_{\mathcal{T}}==_{\mathcal{A}} I_{\mathcal{S}}\end{array}$ \\
\hline $\begin{array}{c}\text { formal cover } \\
\text { convergent cover }+ \\
{ }^{\mathcal{A}}=\wedge^{\mathcal{A}}(\text { weakening and contraction })\end{array}$ & $\begin{array}{c}\text { locale (frame) } \\
\left(\operatorname{Sat}(\mathcal{A}), \bigvee^{\mathcal{A}}, \wedge^{\mathcal{A}}\right)\end{array}$ & $\begin{array}{c}\text { formal cover map = } \\
\text { convergent cover map }+ \\
\quad r^{-} T={ }_{\mathcal{A}} S\end{array}$ \\
\hline
\end{tabular}

\section{Connection with other definitions in the literature}

In this section we review the most relevant different presentations of formal cover (derived from the corresponding versions of formal topology) given in the literature. 
We prove constructively that they all give rise to equivalent categories; this seems to appear here explicitly for the first time. Moreover, we show that they can all be obtained as particular instances of our present definition.

\section{The preorder induced by a basic cover}

For $(S, \triangleleft)$ a basic cover and $a, b \in S$, we consider the preorder (that is, the reflexive and transitive binary relation)

$$
a \leq b \stackrel{\text { def }}{\Longleftrightarrow} a \triangleleft b .
$$

As usual, we write $\downarrow U$ for the subset $\{a \in S:(\exists u \in U)(a \leq u)\}$. Moreover, we put

$$
U \downarrow V=(\downarrow U) \cap(\downarrow V) .
$$

Trivially, we have $a \downarrow b=\mathcal{A} a \cap \mathcal{A} b$ and $U \downarrow V=\bigcup_{a \in U, b \in V} a \downarrow b$ so that $\downarrow$ is determined by its restriction on singletons. The map $U \mapsto \downarrow U$ is a saturation (or closure) operator on $\mathcal{P}(S)$. Thus it makes sense to consider the structure $\left(\operatorname{Sat}(\downarrow), \bigvee^{\downarrow}, \wedge^{\downarrow}\right)$ which is always a lattice. Moreover, all the following hold:

(1) $\downarrow$ is commutative

(2) $\downarrow$ is associative

(3) $\downarrow$ distributes over unions

(4) $\downarrow$-left

(5) contraction

$$
\begin{array}{r}
U \downarrow V=V \downarrow U \\
(U \downarrow V) \downarrow W=U \downarrow(V \downarrow W) \\
U \downarrow\left(\bigcup_{i \in I} V_{i}\right)=\bigcup_{i \in I}\left(U \downarrow V_{i}\right) \\
\mathcal{A}(U \downarrow V) \subseteq \mathcal{A} U \cap \mathcal{A} V \\
U \downarrow U=_{\mathcal{A}} U
\end{array}
$$

for every $U, V, U^{\prime}, V^{\prime} \subseteq S$ and every set-indexed family $\left\{V_{i}\right\}_{i \in I}$ in $\mathcal{P}(S)$. Note, however, that the operation $\downarrow$ does not in general satisfy stability (it does not respect $\left.=_{\mathcal{A}}\right)$, so the triple $(S, \triangleleft, \downarrow)$ is neither a formal cover, nor a convergent cover, nor even a basic cover with operation (definition 2.3).

\section{$5.1 \triangleleft$-formal covers}

Thanks to the discussion above and by proposition 4.3 , it is immediate to see that:

Lemma 5.1 Given a basic cover $(S, \triangleleft)$, the structure $(S, \triangleleft, \downarrow)$ is a formal cover if and only if $\downarrow$ satisfies $\downarrow$-right (in the sense of lemma 4.2).

This justifies the following (see Coquand et al. [7]): 
Definition 5.2 Let $\mathcal{S}=(S, \triangleleft)$ be a basic cover. We say that $\mathcal{S}$ is a $\triangleleft$-formal cover if $(S, \triangleleft, \downarrow)$ is a formal cover, that is, if $a \triangleleft U$ \& $a \triangleleft V \Rightarrow a \triangleleft(U \downarrow V)$ for all $a \in S$ and all $U, V \subseteq S$.

Clearly, $\triangleleft$-formal covers can be identified with those particular formal covers $(S, \triangleleft, \circ)$ for which $\circ=\downarrow$, that is $a \circ b=\{c \in S \mid c \triangleleft a \& c \triangleleft b\}$ for all $a, b \in S$. Thus:

Definition 5.3 We call $\triangleleft$-FCov the full subcategory of FCov whose objects are $\triangleleft$-formal covers.

So a morphism $r$ between two $\triangleleft$-formal covers $(S, \triangleleft)$ and $\left(S^{\prime}, \triangleleft^{\prime}\right)$ is a morphism between the corresponding basic covers which satisfies the extra conditions: $r^{-}\left(a \downarrow^{\prime} b\right)$ $={ }_{\mathcal{A}} r^{-} a \downarrow r^{-} b$ and $r^{-} S^{\prime}={ }_{\mathcal{A}} S$.

Lemma 5.4 If $(S, \triangleleft, \circ)$ is a formal cover, then $\circ$ coincides with $\downarrow$ modulo $=_{\mathcal{A}}$, that is $U \circ V={ }_{\mathcal{A}} U \downarrow V$ for all $U, V \subseteq S$. Hence $(S, \triangleleft)$ is a $\triangleleft$-formal cover and $(S, \triangleleft, \circ)$ is isomorphic to $(S, \triangleleft, \downarrow)$.

Proof To prove the first part of the statement, it is sufficient to check that $a \downarrow b=\mathcal{A}$ $a \circ b$ for all $a, b \in S$. One gets $a \downarrow b \triangleleft a \circ b$ by $\circ$-right and $a \circ b \subseteq a \downarrow b$ by weakening. The second part follows from lemma 4.7 .

This lemma gives immediately that:

Corollary 5.5 The categories $\triangleleft$-FCov and FCov are equivalent.

Finally, one can show that the inclusion functor from FCov to BCov reflects isomorphisms:

Proposition 5.6 Assume that $r: \mathcal{S} \rightarrow \mathcal{S}^{\prime}$ is an isomorphism in BCov with inverse $s$. Then $\mathcal{S}$ is a formal cover if and only if $\mathcal{S}^{\prime}$ is a formal cover. In this case, $r$ and $s$ form an isomorphism also in FCov.

Proof The assumption means that the maps $\mathcal{A} r^{-}$and $\mathcal{A}^{\prime} s^{-}$form a suplattice isomorphism between $\operatorname{Sat}(\mathcal{A})$ and $\operatorname{Sat}\left(\mathcal{A}^{\prime}\right)$, and hence in particular an order-isomorphism. By a general fact of order theory, both $\mathcal{A} r^{-}$and $\mathcal{A}^{\prime} s^{-}$preserve meets, besides joins; in fact, both $\mathcal{A} r^{-}$is left adjoint to $\mathcal{A}^{\prime} s^{-}$and vice versa. So $\operatorname{Sat}(\mathcal{A})$ satisfies distributivity iff so does $\operatorname{Sat}\left(\mathcal{A}^{\prime}\right)$, that is, $\mathcal{S}$ is a formal cover iff so is $\mathcal{S}^{\prime}$. When $\mathcal{S}$ and $\mathcal{S}^{\prime}$ are formal covers, meets are presented by $\downarrow$ and $\downarrow^{\prime}$, that is, $\mathcal{A} U \cap \mathcal{A} V=\mathcal{A}(U \downarrow V)$ and $\mathcal{A}^{\prime} W \cap \mathcal{A}^{\prime} Z=\mathcal{A}^{\prime}(W \downarrow Z)$. It is immediate to see that $\mathcal{A} r^{-}$preserves meets means precisely that $r$ is convergent. Similarly for $s$. 


\section{$5.2 \leq$-formal covers}

The approach via $\triangleleft$-formal covers can appear as the most general since it describes the meet of $\operatorname{Sat}(\mathcal{A})$ in terms of $\downarrow$ and so, in the end, by means of the cover itself. However, it has a serious drawback: to generate $\triangleleft$ inductively one cannot use axioms or rules involving $\downarrow$, at least constructively. In fact, the operation $\downarrow$ is not well-defined until the process of generation of $\triangleleft$ is completed. Thus one has to modify the presentation. One possibility is to approximate the meet by means of a primitive operation $\circ$, as in the present paper, and only at the end of the generation process one sees that $\circ$ and $\downarrow$ coincide (up to $=_{\mathcal{A}}$ ).

Another way to modify the definition of $\triangleleft$-formal cover in order to include inductively generated examples is to use the definition in Coquand [6] and Coquand et al. [7]. The idea is to start from a preordered base and hence to define $\downarrow$ depending on the preorder rather than on $\triangleleft$. The resulting notion is here called a $\leq$-formal cover. The inductive generation of $\leq$-formal covers corresponds to that in Johnstone and Vickers [12] via generators (represented by the preordered set of basic opens) and relations (the starting axiom-set). This approach turned out to be crucial to describe algebraic domains as unary $\leq$-formal covers (see Sambin [28]).

Definition 5.7 $A \leq$-formal cover is a basic cover whose carrier $S$ is equipped with a preorder $\leq$ such that:

$$
\frac{a \leq b \quad b \triangleleft U}{a \triangleleft U} \leq \text {-left } \quad \text { and } \quad \frac{a \triangleleft U \quad a \triangleleft V}{a \triangleleft U \downarrow \leq V} \leq \text {-right }
$$

where: $U \downarrow \leq V=\bigcup_{u \in U, v \in V}(u \downarrow \leq v)$ and $u \downarrow \leq v=\{a \in S \mid a \leq u \& a \leq v\}$.

Clearly, for every $\leq$-formal cover, the structure $(S, \triangleleft, \downarrow \leq)$ is a formal cover. Actually, the original $\leq$-formal cover can be identified with $(S, \triangleleft, \downarrow \leq)$.

Definition 5.8 Let $\leq$-FCov be the full subcategory of FCov whose objects are $\leq$ formal covers.

Every $\triangleleft$-formal cover $(S, \triangleleft, \downarrow)$ is clearly also a $\leq$-formal cover, with $\leq$ defined as in (17). Conversely, every $\leq$-formal cover $(S, \triangleleft, \downarrow \leq)$ is isomorphic to the $\triangleleft$-formal cover $(S, \triangleleft, \downarrow)$ by lemma 5.4. Therefore we can conclude that:

Proposition 5.9 The category $\leq$-FCov is equivalent to $\triangleleft$-FCov and hence to FCov.

Note that the notion of $\leq$-formal cover does not admit a generalization to quantales because convergence expressed via the operation $\downarrow \leq$ already enjoys weakening and contraction. 


\subsection{Formal covers with a monoid operation}

In the original definition by Sambin [26] of formal topology, later revised in [29], convergence was defined by means of a primitive binary operation between basic opens. This approach to pointfree topology turned out to be crucial in order to represent in a predicative way formal topologies on algebraic structures (Schuster [32]), Stone spaces (Ciraulo and Sambin [3] and Negri [23]), Scott domains (Sambin, Valentini and Virgili [31]) and exponentiations between Scott domains (Maietti and Valentini [19]). In the terminology of the present paper, the notion of formal cover in Sambin [29] can be rephrased as:

Definition 5.10 $A \bullet$-formal cover is a formal cover $(S, \triangleleft, \circ)$ together with a binary operation $\bullet: S \times S \rightarrow S$ on elements of $S$ such that $a \circ b=\{a \bullet b\}$ for all $a, b \in S$.

Definition 5.11 We call •-FCov the full subcategory of FCov whose objects are - formal covers.

We are now going to prove that the notion of $\bullet$-formal cover is equivalent to that of $\triangleleft$-formal cover (and hence to all the other notions). We need the following

Lemma 5.12 For every basic cover $\mathcal{S}=(S, \triangleleft)$, there exists a basic cover $\operatorname{Dot}(\mathcal{S})$ such that:

(1) $\operatorname{Dot}(\mathcal{S})$ is isomorphic to $\mathcal{S}$ in BCov;

(2) the carrier of $\operatorname{Dot}(\mathcal{S})$ is naturally endowed with a binary operation $\bullet$;

(3) $\mathcal{S}$ is a $\triangleleft$-formal cover if and only if $\operatorname{Dot}(\mathcal{S})$ is a $\bullet$-formal cover; in that case they are isomorphic as $\triangleleft$-formal covers.

Proof Let $\operatorname{List}(S)$ be the set of all finite lists of elements of $S$. As usual [] is the empty list and $\left[a_{1}, \ldots, a_{n}\right]$ is the list whose elements are $a_{1}, \ldots, a_{n} \in S$. Let $\bullet$ denote concatenation between lists; we extend $\bullet$ also to subsets $K, L \subseteq \operatorname{List}(S)$ in the following way:

$$
K \bullet L=\{k \bullet l \mid k \epsilon K, l \in L\} .
$$

Let $r$ be the relation between $S$ and $\operatorname{List}(S)$ defined by:

$$
\operatorname{br}\left[a_{1}, \ldots, a_{n}\right] \Leftrightarrow\left(b \triangleleft a_{1}\right) \& \cdots \&\left(b \triangleleft a_{n}\right) \quad \text { and } b r[] \text { true }
$$

for all $a_{1}, \ldots, a_{n} \in S$. In other words, we have $r^{-}\left[a_{1}, \ldots, a_{n}\right]=\mathcal{A} a_{1} \cap \ldots \cap \mathcal{A} a_{n}$ $=a_{1} \downarrow \ldots \downarrow a_{n}$ and $r^{-}[]=S$. Finally, let $l \triangleleft^{\prime} K$ be $r^{-} l \triangleleft r^{-} K$, for all $l \in \operatorname{List}(S)$ 
and $K \subseteq \operatorname{List}(S)$. We put $\operatorname{Dot}(\mathcal{S})=\left(\operatorname{List}(S), \triangleleft^{\prime}, \bullet\right)$. This completes the definition of $\operatorname{Dot}(\mathcal{S})$ (and proves 2).

It is easy to check that $\triangleleft^{\prime}$ is a basic cover. By the very definition of $\triangleleft^{\prime}$, the relation $r$ becomes a morphism in BCov from $(S, \triangleleft)$ to $\left(\operatorname{List}(S), \triangleleft^{\prime}\right)$. We check that this is an isomorphism by defining its inverse. Consider the relation $r^{\prime}$ defined by $\left[a_{1}, \ldots, a_{n}\right] r^{\prime} b$ if $a_{1} \downarrow \ldots \downarrow a_{n} \triangleleft b$ and by [ ] $r^{\prime} b$ if $S \triangleleft b$. In other words, for $l \in \operatorname{List}(S)$ and $b \in S$, one has $l r^{\prime} b$ iff $r^{-} l \triangleleft r^{-}[b]$ iff $l \triangleleft^{\prime}[b]$. So $\left(r^{\prime}\right)^{-} b=\mathcal{A}^{\prime}[b]=\mathcal{A}^{\prime}[b]$. Since $r$ is a basic cover map, it follows that $r^{-}\left(r^{\prime}\right)^{-} b={ }_{\mathcal{A}} r^{-}[b]={ }_{\mathcal{A}} b$ and hence $r^{-}\left(r^{\prime}\right)^{-} U={ }_{\mathcal{A}} U$ for all $U \subseteq S$. As a consequence, $r^{\prime}$ is a basic cover map from $\left(\operatorname{List}(S), \triangleleft^{\prime}\right)$ to $(S, \triangleleft)$; in fact, if $a \triangleleft U$, then $r^{-}\left(r^{\prime}\right)^{-} a \triangleleft r^{-}\left(r^{\prime}\right)^{-} U$, that is, $\left(r^{\prime}\right)^{-} a \triangleleft^{\prime}\left(r^{\prime}\right)^{-} U$ by definition of $\triangleleft^{\prime}$. The equation $r^{-}\left(r^{\prime}\right)^{-} b={ }_{\mathcal{A}} b$ also shows that $r^{\prime} r={ }_{\mathbf{B C o v}} i d_{\mathcal{S}}$. It remains to be checked that $r r^{\prime}={ }_{\mathbf{B C o v}} i d_{\operatorname{Dot}(\mathcal{S})}$; for every list $l, r^{-}\left(r^{\prime}\right)^{-} r^{-} l={ }_{\mathcal{A}} r^{-} l$ because $r^{\prime} r={ }_{\mathbf{B C o v}} i d_{\mathcal{S}}$; so $\left(r^{\prime}\right)^{-} r^{-} l=\mathcal{A}^{\prime} l$ by definition of $\mathcal{A}^{\prime}$. Summing up, $r$ is an isomorphism (with inverse $\left.r^{\prime}\right)$ of basic covers. This completes the proof of item 1 .

Because of proposition 5.6, to obtain 3 it is sufficient to show that $\operatorname{Dot}(\mathcal{S})$ is a $\bullet$-formal cover iff $\left(\operatorname{List}(S), \triangleleft^{\prime}\right)$ is a $\triangleleft$-formal cover. One direction follows from lemma 5.4. Conversely, first note that $r^{-}(k \bullet l)=\left(r^{-} k\right) \downarrow\left(r^{-} l\right)$ so that $\bullet$ satisfies weakening, associativity and commutativity. Hence to prove that $\operatorname{Dot}(\mathcal{S})$ is a $\bullet$-formal cover it is sufficient to show that $\bullet$-right holds. To this aim, since $\downarrow^{\prime}$-right holds, it is sufficient to show that $k \downarrow^{\prime} l \triangleleft k \bullet l$. So let $m \epsilon k \downarrow^{\prime} l$, that is, $m \triangleleft^{\prime} k$ and $m \triangleleft^{\prime} l$. This means $r^{-} m \triangleleft r^{-} k$ and $r^{-} m \triangleleft r^{-} l$. So $r^{-} m \triangleleft\left(r^{-} k\right) \downarrow\left(r^{-} l\right)$ since $\mathcal{S}$ satisfies $\downarrow$-right by proposition 5.6. This is precisely $r^{-} m \triangleleft r^{-}(k \bullet l)$, that is, $m \triangleleft^{\prime} k \bullet l$. Hence $k \downarrow^{\prime} l \triangleleft^{\prime}$ $k \bullet l$.

Corollary 5.13 The category •-FCov is equivalent to $\triangleleft-\mathbf{F C o v}$ and hence also to FCov and $\leq$-FCov.

By unfolding the equivalence between $\bullet$-FCov and $\leq$-FCov one can deduce that a --formal cover $(S, \triangleleft, \bullet)$ is identified with the $\leq$-formal cover $(S, \triangleleft, \leq)$ where $a \leq b$ is $a \triangleleft b$. However, in many cases there exists a way to construct a $\leq$-formal cover corresponding to a given $\bullet$-formal cover without using the cover $\triangleleft$ in the definition of $\leq$. For instance, if the operation $\bullet$ on $S$ is associative (and not just associative modulo $={ }_{\mathcal{A}}$ ), then one can define a preorder on $S$ by: $a \leq_{m} b$ if either $a=b$ or $a=l \bullet b$ or $a=b \bullet r$ or $a=l \bullet b \bullet r$ for some $l, r \in S{ }^{4}$ This is the smallest preorder making both $a \bullet b \leq_{m} a$ and $a \bullet b \leq_{m} b$ true. In particular, $a \bullet b \in a \downarrow_{\leq_{m}} b$ and hence $\leq$-right

\footnotetext{
${ }^{4}$ In Battilotti and Sambin [1] a similar definition is given under the further assumption that - is commutative.
} 
follows from the corresponding property for $\bullet$. Moreover, $a \leq_{m} b$ yields $a \triangleleft b$; hence $\leq_{m}$-left holds and so also $a \downarrow_{\leq_{m}} b \triangleleft a \bullet b$. Summing up, $\left(S, \triangleleft, \leq_{m}\right)$ is a $\leq$-formal cover and $a \downarrow_{\leq_{m}} b=\mathcal{A}_{\mathcal{A}} a \bullet b$; hence $(S, \triangleleft, \bullet)$ and $\left(S, \triangleleft, \leq_{m}\right)$ are isomorphic in FCov by lemma 4.7 .

We can also prove that the notions of formal cover presented here are essentially equivalent to the original notion in [26], where the base is required to be a semilattice. There are several ways to see this. For instance, given a $\triangleleft$-formal cover $(S, \triangleleft, \downarrow)$, one can modify the proof of lemma 5.12 by taking $\mathcal{P}_{\omega}(S)$, the set of finite subsets of $S$ (see Ciraulo and Sambin [4]), instead of $\operatorname{List}(S)$ and $\cup$ instead of concatenation. By adapting the definition of the cover in the obvious way, one gets a formal cover $\left(\mathcal{P}_{\omega}(S), \triangleleft^{\prime}, \cup\right)$ which is isomorphic to the given one and, moreover, whose base is a semilattice.

\subsection{Connection with other presentations of quantales}

In [27] the third author introduced the notion of a pretopology in order to give constructive semantics for a class of linear-like logics. The same notion was used in [1] to give a presentation of unital commutative quantales.

In our notation, a pretopology is essentially a unital convergent cover such that $a \circ b$ is a singleton for all $a, b$. So pretopologies form a category, say $\bullet-\mathbf{u C B C o v}$, which is to uCBCov as - -FCov is to FCov. By suitably modifying lemma 5.12 (in particular, by replacing $\downarrow$ with $\circ$ ), it is possible to show that $\bullet$-uCBCov and $\mathbf{u C B C o v}$ are equivalent.

\subsection{Remark on the unary and finitary cases}

When we pass to consider the unary or finitary case, the connection between the different definitions of formal covers changes considerably. Recall that a cover is finitary if for all $a$ and $U$ with $a \triangleleft U$, there exists a finite subset $K$ of $U$ such that $a \triangleleft K$. A finitely cover is unary if the subset $K$ in the definition has at most one element.

For instance, the equivalence between $\leq$-formal covers and $\bullet$-formal covers no longer holds if we restrict to their unary or finitary versions. Indeed, unary $\leq$-formal covers are presentations of algebraic domains while unary •-formal covers present Scott domains (see Sambin [28] and Sambin, Valentini and Virgili [31]). The formal-topological presentation of such classes of domains allows to see why Scott domains are closed under exponentiation whilst algebraic domains are not. Indeed, looking at the construction 
in Maietti and Valentini [20] of the exponential object from an algebraic domain to an inductively generated formal cover (in the category of inductively generated formal covers), one can see that the exponentiation of two algebraic domains is not an algebraic domain, in general.

Concerning the finitary case, we know that Stone spaces correspond to finitary $\bullet$-formal covers (see Negri [23] and Ciraulo and Sambin [3]). Similar characterizations of the finitary versions of the other presentations are still unknown. In particular, it is not clear what structures finitary $\triangleleft$-formal covers represent.

\section{Categorical reading of inductive generation}

In this section, we provide a categorical analysis of our modular method for generating basic covers, convergent covers and formal covers as in sections 1.2, 3.2 and 4.2. We clarify in what sense the generation of a formal cover or of a convergent cover from a given axiom-set is free. This requires finding suitable categories in such a way that our inductive generation processes provide object parts of right adjoints to suitable forgetful functors (right adjoints become left adjoints, as expected, if one works in the opposite categories following the direction of frame maps).

There exists a well known construction of the free frame over a suplattice as a consequence of Johnstone's coverage theorem [10] (see also Vickers [33, theorem 4.4.2]). Here we consider this construction in the corresponding dual categories, and hence we speak of the (co)free formal cover over an inductively generated basic cover. We decompose it into three steps as follows.

- First, we use the base $S$ of the given basic cover to construct a new inductively generated basic cover $O(S)$, with different base and axiom-set, naturally equipped with a pre-convergence operation on subsets and a distinguished subset (that will become the convergence operation and the unit of a quantale, respectively, in a later step).

- Then, as explained in proposition 3.11, we localize the axiom-set of $O(S)$ and generate a unital convergent cover, that is a unital commutative quantale.

- Finally, we add the axioms of weakening and contraction (see proposition 4.9) thus obtaining a formal cover.

These three steps give rise to three adjunctions that are all in the form of a right adjoint to a forgetful functor $U$; the object part of each right adjoint is provided by one of our 
methods of inductive generation. ${ }^{5}$

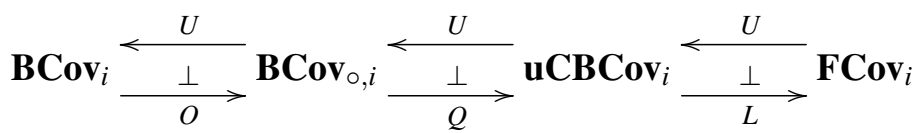

The novelty of our decomposition lies in introducing a subcategory $\mathbf{B C o v} \circ$ of $\mathbf{B C o v}$ whose objects include those built in the first step of the above generation process. The objects of $\mathbf{B C o v}_{\circ}$, called o-basic covers, are basic covers equipped with an operation on subsets of the base that distributes over unions, is associative, commutative and has a unit. This we call a pre-convergence operation; in fact, it enjoys all properties of a convergence operation (section 3) but localization. The morphisms of $\mathbf{B C o v} \circ$ are basic cover maps preserving the pre-convergence operation and the units. ०-basic covers represent the starting data from which we can generate a formal or convergent cover. By using $\mathbf{B C o v}$ 。 we are able to recognize our inductively generated formal covers and convergent covers as free structures. Indeed, in the generation of formal covers, the category $\mathbf{B C o v}$ 。 plays a role analogous to that played by semilattices in Johnstone's coverage theorem.

As we will see, the functor $Q$ is a categorical rendering of proposition 3.11 and lemma 3.14. A similar remark applies to the functor $\mathrm{L}$ with respect to proposition 4.9 and lemma 4.11. The object part of the composition $L \cdot Q \cdot O$ coincides impredicatively with the construction of the free frame over a suplattice. Impredicatively, the functor $Q \cdot L$ is surjective on objects since every locale is co-freely generated from a $\circ$-basic cover. On the contrary, the functor $L \cdot Q \cdot O$ is not surjective on objects, since not every frame is free over some suplattice.

Now we start by introducing the category of o-basic covers:

Definition 6.1 A ०-basic cover $\mathcal{S}=(S, \triangleleft, \circ)$ is a basic cover $(S, \triangleleft)$ with an operation $\circ$ on subsets of $S$ such that

- $\circ$ is distributive over unions modulo $=_{\mathcal{A}}$, that is, $\bigcup_{i \in I}\left(U_{i} \circ V\right)={ }_{\mathcal{A}}\left(\bigcup_{i \in I} U_{i}\right) \circ V$;

- $\circ$ is associative modulo $=_{\mathcal{A}}$, that is, $(U \circ V) \circ W=\mathcal{A} U \circ(V \circ W)$;

- $\circ$ is commutative modulo $=_{\mathcal{A}}$, that is, $U \circ V={ }_{\mathcal{A}} V \circ U$;

- there exists a unit, that is, a subset I such that $a={ }_{\mathcal{A}} a \circ I$ for all $a \in S$.

\footnotetext{
${ }^{5}$ A similar decomposition holds also by taking the non commutative case of $\circ$-basic covers and unital convergent covers, that is, unital (not necessarily commutative) quantales.
} 
Given two o-basic covers $\mathcal{S}$ and $\mathcal{T}$, a $\circ$-basic cover map from $\mathcal{S}$ to $\mathcal{T}$ is a basic cover map $r:\left(S, \triangleleft_{\mathcal{S}}\right) \longrightarrow\left(T, \triangleleft_{\mathcal{T}}\right)$ preserving operation and unit as follows:

$$
r^{-}\left(U \circ_{\mathcal{T}} V\right)=_{\mathcal{A}}\left(r^{-} U\right) \circ_{\mathcal{S}}\left(r^{-} V\right) \quad \text { and } \quad r^{-} I_{\mathcal{T}}={ }_{\mathcal{A}} I_{\mathcal{S}}
$$

for all $U, V \subseteq T$.

It is easy to check that $\circ$-basic covers and $\circ$-basic cover maps form a category. We say that a $\circ$-basic cover is inductively generated when so is its underlying basic cover.

Definition 6.2 We call $\mathbf{B C o v} \circ$ the category of $\circ$-basic covers with $\circ$-basic cover maps. $\mathbf{B C o v}_{\circ, i}$ is the full subcategory of $\mathbf{B C o v} \circ$ whose objects are inductively generated.

These subcategories of basic covers are not relevant per se, in the sense that they do not correspond to specific algebraic structures. Their role is just that of explaining the universal property of inductive generation for formal covers.

Here we prove that there exists a functor $O$, from the category of inductively generated basic covers to its subcategory of o-basic covers, that is right adjoint to the corresponding forgetful functor. This means that we can build the (co)free $\circ$-basic cover generated from a basic cover:

Proposition 6.3 The forgetful functor from $\mathbf{B} \mathbf{C o v}_{\circ, i}$ to $\mathbf{B C o v}_{i}$ has a right adjoint

$$
O: \mathbf{B C o v}_{i} \longrightarrow \mathbf{B C o v}_{\circ, i} \text {. }
$$

Proof Let $\mathcal{S}$ be a basic cover inductively generated by an axiom-set $I, C$. To define the (co)free o-basic cover over $\mathcal{S}$, called $O(\mathcal{S})$, we start from the base $\operatorname{List}(S)$. For $l, k \in \operatorname{List}(S)$, let $l \circ_{O(\mathcal{S})} k$ be (the singleton whose element is) $l \bullet k$, the concatenation of $l$ and $k$. Let the unit $I_{O(\mathcal{S})}$ be (the singleton whose element is) [ ], the empty list. Then the cover of $O(\mathcal{S})$ is the least basic cover $\triangleleft_{O(\mathcal{S})}$ such that:

- $[a] \triangleleft_{O(\mathcal{S})}\{[u] \mid u \in C(a, i)\}$ holds for every $a \in S$ and $i \in I(a)$ (where [b], for $b \in S$, denotes the list of length one whose element is $b$ );

- $l \bullet k \triangleleft O(\mathcal{S}) k \bullet l$ for all $l, k \in \operatorname{List}(S)$;

(associativity, as well as $l=\mathcal{A}_{O(\mathcal{S})} l \bullet[]$, holds automatically).

We need to show that, for every inductively generated basic cover $\mathcal{S}$, a morphism $i_{\mathcal{S}}$ : $O(\mathcal{S}) \longrightarrow \mathcal{S}$ exists in $\mathbf{B C o v}_{i}$ such that, for every $r: \mathcal{T} \longrightarrow \mathcal{S}$ in $\mathbf{B C o v}_{i}$ with $\mathcal{T}$ an 
inductively generated $\circ$-basic cover, there exists a unique $\circ$-basic cover map $\widehat{r}$ such that the following diagram in $\mathbf{B} \mathbf{C o v}_{i}$ commutes.

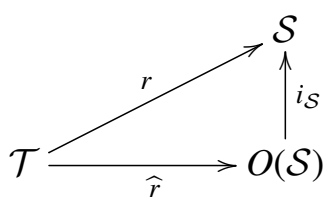

Let $i_{\mathcal{S}}$ be the relation between $\operatorname{List}(S)$ and $S$ defined by $i_{\mathcal{S}}{ }^{-} a=\{[a]\}$. This is a basic cover map

$$
i_{\mathcal{S}}:\left(\operatorname{List}(S), \triangleleft_{O(\mathcal{S})}\right) \rightarrow\left(S, \triangleleft_{\mathcal{S}}\right)
$$

by lemma 1.6; indeed, $i_{\mathcal{S}}^{-} a \triangleleft_{O(\mathcal{S})} i_{\mathcal{S}}{ }^{-} C(a, i)$ holds by the definitions of $\triangleleft_{O(\mathcal{S})}$ and $i_{\mathcal{S}}$. To complete the proof it is sufficient to give the definition of $\widehat{r}$, which is actually compulsory. In fact, $\widehat{r}^{-}[a]=\mathcal{A}_{\mathcal{T}} r^{-} a$ because the diagram must commute, $\widehat{r}^{-}[]=\mathcal{A}_{\mathcal{T}}$ $I_{\mathcal{S}}$ because $\widehat{r}$ must preserve units and finally $\widehat{r}^{-}\left[a_{1}, \ldots, a_{n}\right]=\widehat{r}^{-}\left(\left[a_{1}\right] \bullet \ldots \bullet\left[a_{n}\right]\right)=\mathcal{T}$ $\widehat{r}^{-}\left[a_{1}\right] \circ \ldots \circ \widehat{r}^{-}\left[a_{n}\right]=\mathcal{T} r^{-} a_{1} \circ \ldots \circ r^{-} a_{n}$ because $\widehat{r}$ must respect convergence. It remains to be proved that $\widehat{r}$ is a basic cover map. Since $O(\mathcal{S})$ is inductively generated, it is sufficient to check that $\widehat{r}^{-}[a] \triangleleft \mathcal{T} \widehat{r}^{-}\{[b] \mid b \in C(a, i)\}$, that is, $r^{-} a \triangleleft_{\mathcal{T}} r^{-} C(a, i)$ which holds because $r$ is a basic cover map.

The right adjoint in the previous proposition represents the first step to build the co-free formal cover generated from a basic cover. The second step is to add the axioms making $O(\mathcal{S})$ a unital convergent cover (unital commutative quantale). Also this step enjoys a universal property, namely there exists a functor $Q$, from $\mathbf{B C o v}_{\circ, i}$ to its subcategory $\mathbf{u C B C o v}$ of inductively generated unital convergent covers, that is right adjoint to the corresponding forgetful functor.

Proposition 6.4 The forgetful functor from $\mathbf{u C B C o v}_{i}$ to $\mathbf{B C o v}_{\circ, i}$ has a right adjoint

$$
Q: \mathbf{B C o v}_{\circ, i} \longrightarrow \mathbf{u C B C o v}_{i}
$$

that is surjective on objects.

Proof Let $\mathcal{S}$ be a $\circ$-basic cover inductively generated by an axiom-set $I, C$. We call $Q(\mathcal{S})$ the unital convergent cover generated from the same axiom-set, the same o and the same unit of $\mathcal{S}$ as described in proposition 3.11 and the remark following it. We need to show that for every inductively generated o-basic cover $\mathcal{S}$ there exists a morphism $j_{\mathcal{S}}: Q(\mathcal{S}) \longrightarrow \mathcal{S}$ in $\mathbf{B C o v}_{\circ, i}$ such that, for every $r: \mathcal{T} \longrightarrow \mathcal{S}$ in $\mathbf{B C o v}_{\circ, i}$ 
with $\mathcal{T}$ a unital convergent cover, there exists a unique unital convergent cover map $\widetilde{r}$ such that the following diagram in $\mathbf{B C o v}, i$ commutes.

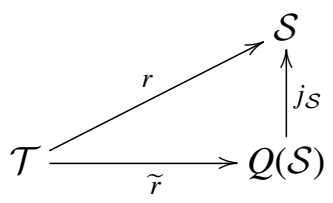

Let $j_{\mathcal{S}}$ be the identity relation on the set $S$. This is a o-basic cover map from $Q(\mathcal{S})$ to $\mathcal{S}$. In fact $a \triangleleft_{Q(\mathcal{S})} C(a, i)$ holds for all $a \in S$ and $i \in I(a)$, because $Q(\mathcal{S})$ is generated by an axiom-set extending $I, C$. Moreover, $j_{\mathcal{S}}$ respects convergence and units since $Q(\mathcal{S})$ has the same $\circ$ and unit of $\mathcal{S}$. To complete the proof it is sufficient to define $\widetilde{r}$ as $r$ itself given that $r$ is also a map toward $Q(\mathcal{S})$ by lemma 3.14 .

Since every inductively generated unital convergent cover can be obtained as in proposition 3.11 we conclude that $Q$ is surjective.

The above proposition is a refinement of the universal property of quantale presentations in Battilotti and Sambin [1]. In fact, the authors of [1] work with a monoid operation on the base. Hence their result corresponds to the existence of a right adjoint, from the subcategory $\mathbf{B C o v}{ }_{\bullet}, i$ (see below for a precise definition) of $\mathbf{B} \mathbf{C o v}_{\circ}, i$, that is just a restriction of our functor $Q$.

The last step is the generation of a formal cover from an inductively generated unital convergent cover.

Proposition 6.5 The forgetful functor from $\mathbf{F C o v}$ to $\mathbf{u C B C o v} i$ has a right adjoint

$$
L: \mathbf{u C B C o v}_{i} \longrightarrow \mathrm{FCov}_{i}
$$

that is surjective on objects.

Proof Let $\mathcal{S}$ be a convergent cover with unit inductively generated by an axiom-set $I, C$. We call $L(\mathcal{S})$ the formal cover generated from the same $I, C$ and the same $\circ$ of $\mathcal{S}$ as in proposition 4.9. We show that there exists a morphism $k_{\mathcal{S}}: L(\mathcal{S}) \longrightarrow \mathcal{S}$ in $\mathbf{u C B C o v}_{i}$ such that, for every $r: \mathcal{T} \longrightarrow \mathcal{S}$ in $\mathbf{u C B C o v}_{i}$ with $\mathcal{T}$ a formal cover, there exists a unique continuous map $\bar{r}$ such that the following diagram in $\mathbf{u C B C o v} i$ commutes.

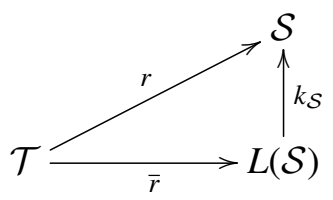


Let $k_{\mathcal{S}}$ be the identity relation on the set $S$. This is a unital convergent cover map from $L(\mathcal{S})$ to $\mathcal{S}$ by lemma 3.14. In fact $a \triangleleft_{L(\mathcal{S})} C(a, i)$ holds for all $a \in S$ and $i \in I(a)$, because $L(\mathcal{S})$ is generated by an axiom-set extending $I, C$. Moreover, $k_{\mathcal{S}}$ trivially respects $\circ$ and units. Finally, we define $\bar{r}$ as $r$ itself, since $r$ is also a map into $L(\mathcal{S})$ by lemma 4.11 .

Since every inductively generated formal cover can be obtained from an inductively generated unital convergent cover by adding the axioms of weakening and contraction, we conclude that $L$ is surjective.

The functors $O, Q$ and $L$ give a decomposition of the right adjoint of the forgetful functor from formal covers to basic covers:

Corollary 6.6 The functor $L \cdot Q \cdot O: \mathbf{B C o v}_{i} \longrightarrow \mathbf{F C o v}_{i}$ is a right adjoint to the forgetful functor from $\mathbf{F C o v}_{i}$ to $\mathbf{B C o v}_{i}$.

This is a predicative counterpart of the existence of a left adjoint to the forgetful functor from the category of frames to that of suplattices. If $\mathcal{S}$ already is a formal cover, that is $\operatorname{Sat}(\mathcal{A})$ is a locale, then the construction $L \cdot Q \cdot O(\mathcal{S})$ gives a predicative presentation of what is known as the lower power locale (see Vickers [33]) of $\operatorname{Sat}(\mathcal{A})$.

From propositions 6.4 and 6.5, we conclude:

Corollary 6.7 The functor $L \cdot Q: \mathbf{B C o v}_{\circ, i} \longrightarrow \mathbf{F C o v}_{i}$ is right adjoint to the forgetful functor from $\mathbf{F C o v}{ }_{i}$ to $\mathbf{B C o v}_{\circ, i}$ and, moreover, is surjective on objects.

This result shows that the method of inductively generating formal covers from an axiom-set enjoys a universal property with respect to the category $\mathbf{B C o v} \mathbf{o v}_{\circ}$. Besides the functor $L \cdot Q$ presented here, there are in the literature other ways for generating a formal cover given some initial data. We need to recall three such methods to be able to explain how our approach is a refinement of them all.

In Coquand et al. [7] it is shown how to generate a $\leq$-formal cover starting from an axiom-set on a preordered set $(S, \leq)$. One can easily check that, in the specific case in which $\delta(a \circ b)=a \downarrow \leq b$, our rules to generate formal covers (proposition 4.9) become perfectly equivalent to those given in [7]. We now can see that the construction in [7] is co-free with respect to a suitable subcategory of $\mathbf{B C o v}_{\circ, i}$.

Definition 6.8 We call $\mathbf{B C o v} \leq$ the full subcategory of $\mathbf{B C o v}$ 。whose objects satisfy $\circ=\downarrow \leq$ for some preorder $\leq$ on the base. $\mathbf{B C o v}_{\leq, i}$ is its full subcategory whose objects are inductively generated. 
As a consequence of the results above, we have:

Corollary 6.9 The functor $L \cdot Q$ restricts to a functor from $\mathbf{B C o v}_{\leq, i}$ to the category $\leq-$ FCov $_{i}$ of inductively generated $\leq-$ formal covers. This restriction is right adjoint to the forgetful functor in the opposite direction and, moreover, is surjective on objects.

This adjunction explains in what sense the construction of inductively generated $\leq$ formal covers in [7] is co-free. A direct proof can be obtained by the instantiation of lemma 4.11 to formal covers where $\circ=\downarrow \leq$. This gives precisely the result which has played a key role when dealing with inductively generated $\leq$-formal covers (see for instance [16]). Note, however, that the adjunction between $\mathbf{B C o v} \leq, i$ and $\leq-\mathbf{F C o v} \mathbf{C o v}_{i}$ cannot be decomposed via quantales. In fact, the functor $Q$, when applied to an object in $\mathbf{B C o v}_{\leq, i}$, gives already a $\leq$-formal cover (weakening and contraction automatically hold). In other words, $L \cdot Q$ and $Q$ coincide on $\mathbf{B C o v}_{\leq, i}$.

The second approach we want to recall is that described in Battilotti and Sambin [1]. There a monoid structure is assumed on the base. So the starting data for generating a formal cover can be thought of as objects of the following category.

Definition 6.10 We call BCov • the full subcategory of $\mathbf{B C o v}_{\text {}}$ whose object satisfy $a \circ b=\{a \bullet b\}$ (for all $a, b \in S$ ) for some monoid operation $\bullet$ on the base $S . \mathbf{B C o v}_{\bullet}, i$ is its full subcategory whose objects are inductively generated.

As before, the functor $L \cdot Q$ can be restricted to $\mathbf{B C o v}_{\bullet, i}$ to obtain the following categorical result of the universal property in [1]:

Corollary 6.11 The functor $Q$ restricted to a functor from $\mathbf{B C o v}_{\bullet, i}$ to $\bullet-\mathbf{u C B C o v}$ is a right adjoint to the forgetful functor in the opposite direction.

We finally come to the third approach, namely Johnstone's coverage theorem in [10, 33]. As one can recognize, Johnstone's result reads in our framework just as proposition 4.9 in the case in which $S$ has a $\wedge$-semilattice structure and $a \circ b=\{a \wedge b\}$. Hence the data required by Johnstone's method amount to an inductively generated basic cover whose base has a semilattice structure. So we give the following:

Definition 6.12 We call $\mathbf{B C o v} \wedge$ the full subcategory of $\mathbf{B C o v}_{\diamond}$ whose objects satisfy $a \circ b=\{a \wedge b\}$ (for all $a, b \in S$ ) for some semilattice operation $\wedge$ on the base $S$. $\mathbf{B C o v}_{\wedge, i}$ is its full subcategory whose objects are inductively generated. 
Clearly $\mathbf{B C o v}_{\wedge}$ is a subcategory both of $\mathbf{B C o v}_{\leq}$and of $\mathbf{B C o v}$. . With this notation, Johnstone's result becomes:

Proposition 6.13 The functor $L \cdot Q$ restricted to a functor from $\mathbf{B C o v} \operatorname{Cos}_{\wedge}$ to $\mathbf{F C o v}_{i}$ is right adjoint to the forgetful functor in the opposite direction.

The following diagram summarizes the adjunctions discussed above (each forgetful functor $U$ is a left adjoint) together with the (full) inclusions between the various categories. It is easy to check that all sub-diagrams commute.

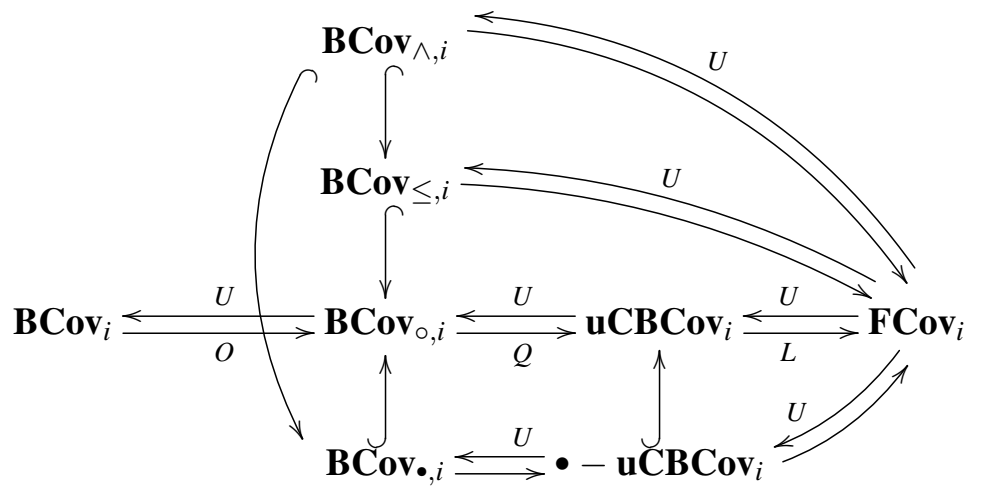

There is also another way to obtain a formal cover from an arbitrary basic cover, which however does not lead to a functor from $\mathbf{B C o v} i$ to $\mathbf{F} \mathbf{C o v}_{i}$. This construction starts from a basic cover $\mathcal{S}=(S, \triangleleft)$ generated from an axiom-set $I, C$ and applies the method of proposition 4.9 with $\delta(a, b)=a \downarrow b$, where $\downarrow$ is defined through $\triangleleft$ itself as in (17) and (18). No doubt, this method produces a formal cover, namely $L \cdot Q(S, \triangleleft, \downarrow)$. However, it cannot be extended to a functor from $\mathbf{B C o v}_{i}$ to $\mathbf{F C o v}_{i}$ since there is no reason for a basic cover map to respect the operation $\circ=\downarrow$. Note also that the formal cover $L \cdot Q(S, \triangleleft, \downarrow)$ is quite different from $L \cdot Q \cdot O(S, \triangleleft)$. This is well visible when $\mathcal{S}$ itself is a $\triangleleft$-formal cover. In fact, in this case $L \cdot Q(S, \triangleleft, \downarrow)$ is $(S, \triangleleft, \downarrow)$ itself, while $L \cdot Q \cdot O(\mathcal{S})$ presents the lower power locale of $\operatorname{Sat}(\mathcal{A})$ and hence it is not isomorphic to $\mathcal{S}$. In general, one can see that the formal cover $L \cdot Q(S, \triangleleft, \downarrow)$ presents the largest frame contained in the suplattice $\operatorname{Sat}(\mathcal{A})$.

\section{Conclusions}

We have presented a new definition of formal cover/formal topology that generalizes all other definitions given so far. This has been obtained by considering an operation $\circ$ 
between subsets which is uniquely determined by its trace on singletons. Our approach seems to gather all the advantages of previous definitions. It allows us to reach the definition of formal cover in a modular way passing through the case of quantales, as it happens for the approach via a monoid operation on the base (see $\bullet$-formal covers in section 5). At the same time, it provides a uniform method of inductive generation that includes the one originally introduced in [7].

Our new definition of convergent covers and formal covers allows us to reproduce Joyal-Tierney's characterization of quantales and locales [13] in a straightforward way. Previous definitions of formal cover were not apt to this purpose. The definition of $\leq$ formal cover does not generalize to represent quantales. Also the original definition [26] is too specific; it can be generalized as in [27] and [1] to represent quantales, but the only way one can see to obtain Joyal-Tierney's characterization is to pass through the equivalence with the new notion introduced here.

Our new presentation of convergence offers a uniform and modular method for generating formal covers, convergent covers and basic covers inductively. This uniformity allows us to recognize in what sense the various inductive constructions provide free structures and thus refine Johnstone's coverage theorem. Our analysis supplies a neat decomposition of the well known adjunction associated to the free frame over a suplattice. This would hardly be possible with the presentation of formal covers in the literature.

\section{Acknowledgements}

We thank Pino Rosolini for fruitful discussions on Joyal-Tierney's representation of frames as monoids and Steve Vickers for pointing out the connection between free frames over suplattices and lower power locales.

\section{References}

[1] G. Battilotti and G. Sambin, Pretopologies and a uniform presentation of sup-lattices, quantales and frames, Annals of Pure and Applied Logic, 137(2006), 30-61, doi: 10.1016/j.apal.2005.05.017.

[2] F. Ciraulo, M. E. Maietti and P. Toto, Constructive version of Boolean algebra, Logic Journal of the IGPL 21 (2013), 44-62, doi: 10.1093/jigpal/jzs021.

[3] F. Ciraulo and G. Sambin, Finitary formal topologies and Stone's representation theorem, Theoretical Computer Science 405 (2008), 11-23, doi: 10.1016/j.tcs.2008.06.020. 
[4] F. Ciraulo and G. Sambin, Finiteness in a minimalist foundation, Lecture Notes in Computer Science 4941(2008), 51-68, doi: 10.1007/978-3-540-68103-8_4.

[5] F. Ciraulo and G. Sambin, The overlap algebra of regular opens, Journal of Pure and Applied Algebra 214 (2010), 1988-1995. doi: 10.1016/j.jpaa.2010.02.002.

[6] T. Coquand, Formal topology with posets; preprint available at www.cse.chalmers . se/ coquand/alt.ps.

[7] T. Coquand, G. Sambin, J. Smith and S. Valentini, Inductively generated formal topologies, Annals of Pure and Applied Logic 124 (2003), 71-106, doi: 10.1016/S01680072(03)00052-6.

[8] G. Curi, On the existence of Stone-Čech compactification, Journal of Symbolic Logic 75 (2010), 1137-1146, doi: 10.2178/js1/1286198140.

[9] M. P. Fourman and R. J. Grayson, Formal spaces, The L. E. J. Brouwer Centenary Symposium, Studies in Logic and the Foundations of Mathematics 110, North-Holland, Amsterdam, 1982, 107-122, doi: 10.1016/S0049-237X(09)70126-0.

[10] P. T. Johnstone, Stone Spaces, Cambridge Studies in Advanced Mathematics 3, Cambridge University Press, 1982.

[11] P. T. Johnstone, The point of pointless topology, Bulletin of the American Mathematical Society (N.S.), 8 (1983), 41-53, doi: 10.1090/S0273-0979-1983-15080-2.

[12] P. Johnstone and S. J. Vickers, Preframe presentations present, in A. Carboni, M. C. Pedicchio and G. Rosolini (eds.) Category Theory - Proceedings, Como 1990, Springer Lecture Notes in Mathematics 1488 (1991), 193-212, doi: 10.1007/BFb0084221.

[13] A. Joyal and M. Tierney, An Extension of the Galois Theory of Grothendieck, Memoirs of the American Mathematical Society 51, 1984.

[14] G. M. Kelly, On MacLane's conditions for coherence of natural associativities, commutativities, etc., Journal of Algebra 1 (1964), 397-402.

[15] S. Mac Lane, Categories for the Working Mathematician, Springer-Verlag, New York, 1998 (2nd ed.).

[16] M. E. Maietti, Predicative exponentiation of locally compact formal topologies over inductively generated ones, in L. Crosilla and P. Schuster (eds.), From Sets and Types to Topology and Analysis: Practicable Foundation for Constructive Mathematics, Oxford Logic Guides 48, 202-222, Oxford University Press, 2005, doi: 10.1093/acprof:oso/9780198566519.003.0013.

[17] M. E. Maietti, A minimalist two-level foundation for constructive mathematics, Annals of Pure and Applied Logic 160 (2009), 319-354, doi: 10.1016/j.apal.2009.01.006.

[18] M. E. Maietti and G. Sambin, Toward a minimalist foundation for constructive mathematics in L. Crosilla and P. Schuster (eds.), From Sets and Types to Topology and Analysis: Practicable Foundation for Constructive Mathematics, Oxford Logic Guides 48, 91-114, Oxford University Press, 2005, doi: 10.1093/acprof:oso/9780198566519.003.0006. 
[19] M. E. Maietti and S. Valentini, Exponentiation of Scott formal topologies, Workshop on Domains VI 2002, Electronic Notes in Theoretical Computer Science 73 (2003), 111-131.

[20] M. E. Maietti and S. Valentini, A structural investigation on formal topology: coreflection of formal covers and exponentiability, Journal of Symbolic Logic 69 (2004), 967-1005, doi: 10.2178/js1/1102022209.

[21] P. Martin-Löf, Notes on Constructive Mathematics, Almqvist \& Wiksell, 1970.

[22] C. J. Mulvey, “\&”, Rendiconti del Circolo Matematico di Palermo, Serie II, Supplemento 12 (1986), 99-104.

[23] S. Negri, Stone bases, alias the constructive content of Stone representation, in Logic and Algebra, A. Ursini and P. Aglianò (eds), 617-636, Dekker, New York, 1996.

[24] S. Negri, Continuous domains as formal spaces, Mathematical Structures in Computer Science 12 (2002), 19-52, doi: 10.1017/S0960129501003450.

[25] K. I. Rosenthal, Quantales and their Applications, Pitman Research Notes in Math. 234, Longman, 1990.

[26] G. Sambin, Intuitionistic formal spaces - a first comunication, in D. Skordev, editor, Mathematical Logic and its Applications, 187-204, Plenum, 1987.

[27] G. Sambin, Pretopologies and completeness proofs, Journal of Symbolic Logic 60 (1995), 861-878, doi: 10.2307/2275761.

[28] G. Sambin, Formal topology and domains, Electronic Notes in Theoretical Computer Science 35 (2000), 1-14.

[29] G. Sambin, Some points in formal topology, Theoretical Computer Science 305 (2003), 347-408, doi: 10.1016/S0304-3975(02)00704-1.

[30] G. Sambin, The Basic Picture and Positive Topology. New structures for Constructive Mathematics, Oxford University Press, to appear.

[31] G. Sambin, S. Valentini and P. Virgili, Constructive domain theory as a branch of intuitionistic pointfree topology, Theoretical Computer Science 159 (1996), 319-341, doi: 10.1016/0304-3975(95)00169-7.

[32] P. M. Schuster, Formal Zariski topology: positivity and points, Annals of Pure and Applied Logic 137 (2006), 317-359, doi: 10.1016/j.apal.2005.05.026.

[33] S. Vickers, Topology via Logic, Cambridge Tracts in Theoretical Computer Science 5, Cambridge University Press, Cambridge, 1989.

Department of Mathematics, The University of Padua

Via Trieste 63, 35121 Padova, Italy

ciraulo@math.unipd.it, maietti@math.unipd.it, sambin@math.unipd.it

http://www.math.unipd.it/ ciraulo, http://www.math.unipd.it/ maietti, http://www.math.unipd.it/ sambin

Received: 12 June 2012 Revised: 25 January 2013

Journal of Logic \& Analysis 5:2 (2013) 